\title{
A Novel Approach for Execution of Distributed Tasks on Mobile Ad Hoc Networks*
}

\author{
P. Basu, W. Ke, and T.D.C. Little \\ Department of Electrical and Computer Engineering, Boston University \\ 8 Saint Mary's St., Boston, MA 02215 \\ $\{$ pbasu,ke,tdcl\}@bu.edu
}

July 22, 2001

MCL Technical Report No. 07-22-2001

\begin{abstract}
Mobile ad hoc networks (MANETs) have received significant attention in the recent past owing to the proliferation in the numbers of tetherless portable devices, and rapid growth in popularity of wireless networking. Most of the MANET research community has remained focused on developing lower layer mechanisms (such as channel access and routing) for making MANETs operational. However, little focus has been applied on higher layer issues, especially application modeling. In this paper, we present a novel distributed application framework based on task graphs that enables a large class of resource discovery based applications on MANETs. A distributed application is represented as a complex task comprised of smaller sub-tasks that need to be performed on different classes of computing devices with specialized roles. Execution of a particular task on a MANET involves several logical patterns of data flow between classes of such specialized devices. These data flow patterns induce dependencies between the different classes of devices that need to cooperate to execute the application. Such dependencies yield a task graph representation of the application.
\end{abstract}

We focus on the problem of executing distributed tasks on a MANET by means of dynamic selection of specific devices that are needed to complete the tasks. In this paper,

${ }^{*}$ This work was supported by the NSF under grant No. ANI-0073843. 
we present simple and efficient algorithms for dynamic discovery and selection of suitable devices in a MANET from among a number of them providing the same functionality. This is carried out with respect to the proposed task graph representation of the application, and we call this process Dynamic Task-based Anycasting. Our algorithm periodically monitors the logical associations between the selected devices, and in the event of a disruption in the application owing to failures in any component in the network, it adapts to the situation and dynamically rediscovers the affected parts of the task graph, if possible. Finally, we propose metrics for evaluating the performance of these algorithms and report simulation results for a variety of application scenarios with different degrees of complexity, device mobility, and transmission range.

\section{Introduction}

With the shrinking size of tetherless computing devices and increasing diversity of their capabilities, the value of pervasive computing is rapidly becoming real. As these devices proliferate in number, exploiting the full potential of a network of such devices while not frustrating the end-user with interminable configuration tasks and wire-plugging exercises proves to be a challenging issue indeed.

Established technologies such as IEEE 802.11 [9] and burgeoning technologies such as Bluetooth [5] are poised to bring true tetherless computing applications to the end user in every possible way. A mobile ad hoc network (MANET) is a rapidly deployable, autonomous system of mobile devices which are connected by wireless links to form an arbitrary graph at any instant of time. With increase in popularity of portable devices and wireless connectivity standards, MANETs are likely to gain popularity in the near future, especially in settings where a networking infrastructure is expensive, cumbersome, or impossible to construct. We can conceive scenarios in which the environment surrounding us consists of a large number of specialized as well as multipurpose devices, many of them portable, and linked through wireless connections, albeit with fluctuating link availability. Ideally, such pervasive networks can enable a broad range of distributed applications that need exchange of information between multiple devices.

When a large number of computing devices become equipped with wireless connectivity, and they form an ad hoc network, they can offer services to other devices for performing several tasks. In such a situation, since the service providing devices may themselves be mobile, a user cannot rely on one particular device for a certain service since its reachabil- 


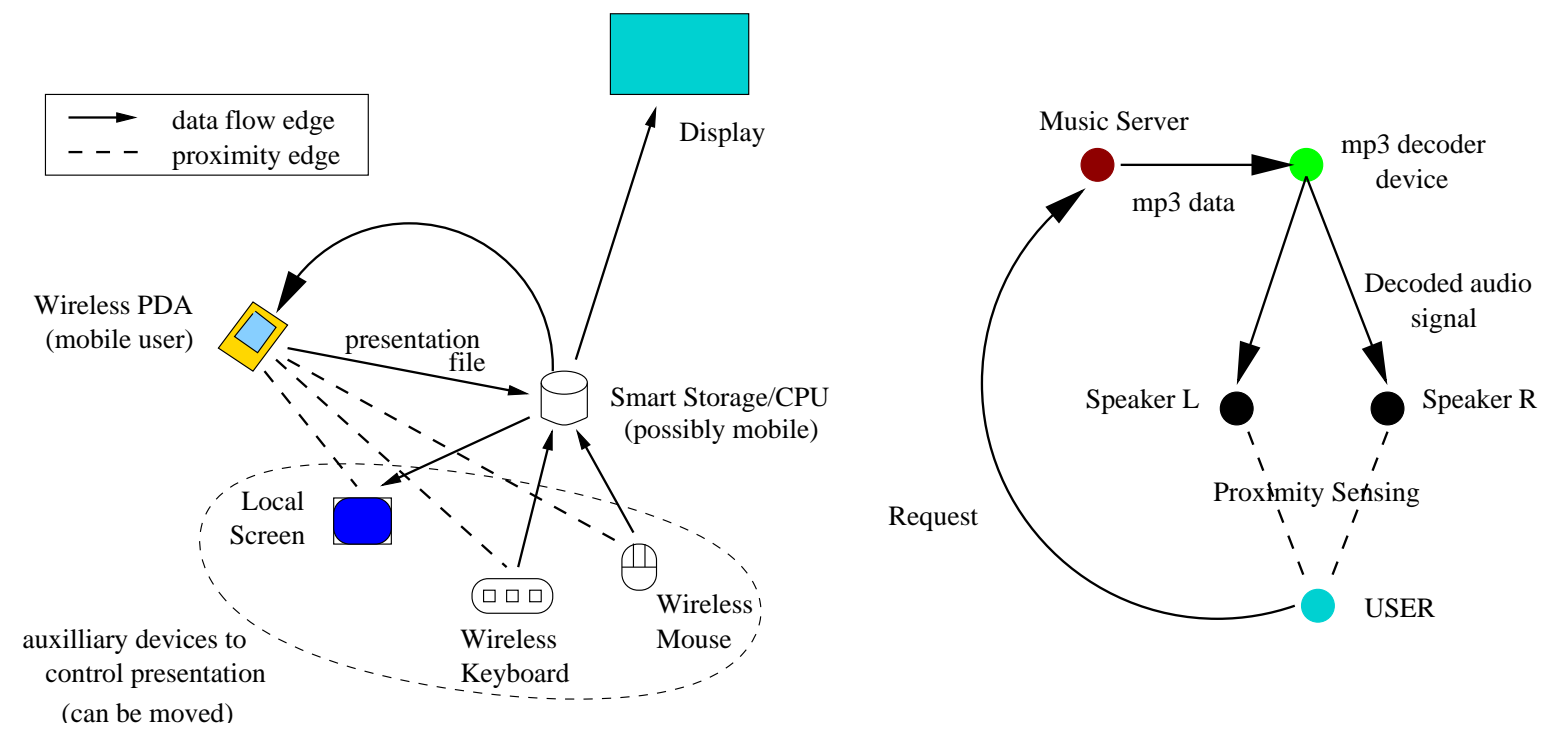

Figure 1: Smart Office and Home Applications: (a) Smart Presentation Task, and (b) Stereo Music Service

ity/availability is not guaranteed. Instead, a user must be prepared to access the required service from any of several devices in the MANET providing similar services, if possible.

Significant previous research focused on lower layer MANET issues such as channel access $[18,4]$ and routing $[17,20,15]$. However, higher layer issues related to application design have received little attention in this context. We bridge this gap with a novel scheme for modeling and executing distributed applications on MANETs that rely on services offered by other devices scattered across the network.

We introduce the abstraction of a Task-Graph (TG) for representing higher-level tasks (or applications) that a user may want to perform, in terms of smaller sub-tasks. It is a graph composed of nodes and edges, where the nodes represent the classes $^{1}$ of devices/services needed for processing data related to the task while the edges represent necessary associations between different nodes for performing the task. Fig. 1 shows two examples of task graphs in the pervasive computing domain. Fig. 1(a) shows the TG of a smart presentation application, in which a user, carrying only a PDA with wireless connectivity capacities, automatically finds and instantiates the devices in a room needed to make a full presentation. Fig. 1(b) shows the TG of a music service application, in which the application tracks the physical location of the user and instantiates the appropriate speaker devices that will play the music the user has selected. Both applications are mentioned in further detail in the next section.

Thus when a task is to be executed, specific devices are selected (in other words,

\footnotetext{
${ }^{1}$ Printer, Photocopier, Digital Picture Frame etc. are examples of classes
} 
instantiated) at runtime, and are made to communicate with one another according to the specifications of the TG. More specifically, for each class of device in TG, one suitable instance needs to be chosen to take part in task execution. We call this process Task-based Anycasting or Embedding. The adjective dynamic is added since the choice of device instances may change with time due to mobility.

When a participating device becomes unavailable, a new substitute device with similar capabilities is selected to continue the task. The TG abstraction of a distributed task is advantageous in many ways. It is inherently distributed, as most pervasive applications and services of the future are likely to be, since more and more specialized devices will need to communicate with one another to offer more and more powerful services. It also offers hierarchical composability, as collections of devices can be logically grouped together to constitute a single node in a TG.

Although we do not prove it explicitly, we argue that optimal embedding of a general graph onto a general MANET is a hard problem. However, if TG is a tree, we show that it is possible to perform optimal embedding in polynomial time, and present one such algorithm. But since the running time of this algorithm is high and it assumes knowledge of the entire topology, we present a simpler and practical distributed algorithm for dynamic instantiation of TGs on a MANET for executing the application corresponding to the TG. In the event of a disruption in the application owing to failures in any component in the network, our algorithm quickly adapts to the situation and dynamically re-instantiates the affected parts of the TG.

We measure the success of our distributed instantiation algorithm by certain metrics such as average time for instantiation, quality of the instantiation (in this paper, the technical term used for this is dilation), frequency of disruption of tasks due to mobility or route failures, and average effective throughput that is achieved after application data transmission begins.

The rest of the paper is organized as follows: Section 3 introduces example scenarios that motivate research in our Task-Graph approach. Section 4 introduces the theoretical foundations for the TG concept. Section 2 briefly describes the related work in the literature. Section 5 presents our distributed algorithm for TG instantiation in a network. Section 6 presents simulation results of the algorithm presented. Section 7 concludes the paper. 


\section{Related Work}

In recent years, service discovery in networks has been a popular topic of research in the industry as exemplified by SLP [13] and Sun's Jini [21]. In both these schemes, a service providing computer registers itself with its attributes at a centralized directory server which the clients can lookup on demand. MOCA is a variation of Jini without any centralized registry [3]. It is specifically designed for mobile computing devices - every device has a service registry component which only the applications running on the local and surrounding devices can benefit from. Our approach is different from these as it operates at a logical layer above service discovery and it can co-exist with any of these schemes. Also, it does not depend upon any centralized directory service.

Some researchers have proposed to capture user-intent for discovering appropriate devices suitable to them. Portolano project [10] emphasizes the use of data centric networking which is really a low-level implementation technique that can potentially have TG as a higher-level abstraction. In INS [1], the user intent is abstracted into collections of attributevalue pairs that describe what the user wants. The specific devices that will perform the desired service will be selected by special entities called Intentional Name Resolvers. INS has a feature called Intentional Anycast and late binding which are similar to what we call instantiation of TG nodes. However, it does not to attempt to systematically represent the relationships between the components of a distributed task, which is our principal focus. Hodes et al. [16] have investigated means of composing services for heterogeneous mobile clients. Their work primarily focuses on controlling office equipment from mobile devices and design of client-device interfaces. They too have not addressed the issues involved in composing complex services from simple devices with specific interaction patterns between them.

IBM's PIMA has a vision somewhat similar to ours. In their vision paper [2], they argue very briefly for the design of applications in terms of sub-tasks instead of specific devices. However, they have not mentioned any approach for realizing this vision so far. Our task-graph concept on the other hand is a systematic and concrete approach which can help realize this vision.

The concept of a task graph was originally used in parallel computing and scheduling literature for representing tasks that can be split temporally into sub-tasks and then allocated to different homogeneous processors connected by a fixed high-performance interconnect for reducing the total completion time $[6,19]$. Our notion of a task graph is different from 
this classical one. We are not necessarily concerned with tasks that are distributable among multiple homogeneous processors for speed-up. Rather, most tasks that we are concerned with in this work involve several specialized heterogeneous devices that communicate with each other, and there is no notion of minimizing the total completion time. However, if we are interested in solving a large scale distributed computing task on a network of homogeneous mobile devices, then our notion of a task graph will be similar to the classical one. Therefore, our task graph formulation is more general than the one used in the parallel computing context. We do not discuss the homogeneous device scenario in this paper. To the best of our knowledge, this is the first attempt towards modeling distributed applications on a MANET using task graphs.

\section{Examples of Application Scenarios}

In this section we describe a few application scenarios which motivated our research. We give examples from smart offices and homes, disaster relief, and sensor networks.

Smart Pervasive Computing Applications Here we consider an application for future smart offices, conference halls, or ad-hoc meeting rooms. Imagine a large hall with a number of display screens, projectors, wireless keyboards and pointing devices. Also assume that there are a number of smart storage devices which can run popular file-viewing software. These devices can store a presentation file in their local storage and can render them on a display screen using the appropriate software ${ }^{2}$. Devices are tetherless and therefore can be moved around in the room freely. The presenter carries only a PDA (with wireless connectivity) that contains the presentation file. The file, or portions of it, are transferred from the PDA to a suitable smart storage device depending on the type of presentation software and other factors to be outlined later. The presenter will need a pointing device, a keyboard, perhaps, and a local screen to control the presentation, but does not care about which devices actually perform the presentation task.

Fig. 1(a) depicts the presentation task with a graph where the vertices stand for categories of devices involved in the task and the edges stand for data flow between device categories or other factors like physical proximity. Assuming that there is no infrastructure support in the hall, a multi-hop ad-hoc network is formed by these devices among each other.

\footnotetext{
${ }^{2}$ Laptops of audience members with wireless connectivity can easily take up this role.
} 
In this scenario, a self-organizing protocol is required to discover the most suitable display screen, smart storage device, keyboard, mouse and local screen with respect to the presenter's location and data requirements. The presenter can move around in the hall either naturally or for displaying multiple items at the same time, and a different set of peripherals may need to be discovered for optimal effect. Note that the nearest smart storage device containing the suitable viewer may not be the best one for the nearest keyboard and the nearest display to communicate with. The discovery process must consider the relationships between vertices in the corresponding task graph. Thus the presentation task can be achieved seamlessly without bothering the user with configuring the chosen peripheral devices.

Fig. 1(b) illustrates an example of an application for future smart homes. Imagine a user wearing a proximity sensor embedded in his/her shirt roaming around in the house while listening to music. When he/she requests a particular song from a music server (hosted in the house or somewhere in the Internet), the music server streams the mp3 file to a suitable mp3 decoder in the house which may be a hardware player or a software decoder residing on a PC. The proximity sensor in the users shirt senses the nearest set of speakers, and the selected mp3 decoder device starts streaming the audio to the selected set of speakers. If the user moves to another room, the music follows the user automatically since a new set of devices is dynamically selected, triggered by the proximity sensor.

Scalable Coordination in Disaster Relief In disaster relief situations involving earthquakes, fires or floods, there are various teams of workers involved such as law enforcement officers, fire fighters, rubble removers, rescue workers, paramedics, and ambulance personnel, among others. Each of these groups of people have specialized roles in the rescue operations, and they are expected to be in constant touch with each other through their personal handheld devices. However, usually all rescue workers do not need to communicate with all others. For example, the fire fighters receive data from sensors and communicate with paramedics, which in turn need to communicate with the ambulance personnel only. Hence, one can establish certain communication patterns between different types of rescue workers such that only messages that are relevant to them arrive at their PDAs.

Therefore, instead of forming one huge multicast tree, one can form a number of smaller multicast groups (each corresponding to a team of workers) connected together by a small anycast tree. The nodes in the anycast tree are similar to the nodes in an embedded task graph, each belonging to a specialized type defined by their roles, e.g. Police, Ambulance etc. An edge in an anycast tree joins one member of a specialized team to a member 
of another specialized team, if those particular teams need to communicate. e.g., if fire fighters need to communicate with paramedics, one fire fighter's PDA acts as the gateway of communication, and so does one paramedic's PDA. Communicating along the anycast tree could result in more scalable management of resources than communicating via pure multicast which could waste a significant amount of bandwidth. Our instantiation algorithms can efficiently establish an anycast tree along which such communication can occur. If the selected anycast nodes are lost due to network partitions, then replacement nodes can be selected dynamically.

Smart Sensor Networks Here the higher-level task is to sense and track a given mobile object using a network of embedded sensors. Suppose that for a desired level of fidelity, data from four sensors is needed at any time instant. The task here translates to discovering four sensors out of the sea of networked sensors that will optimally track a given object. An object triangulation example has been explained in greater detail in reference [11].

\section{A Task Graph Based Modeling Framework}

The use of graph theoretic approaches is well known in traditional parallel and distributed computing systems $[6,19]$. We believe that it can be a promising and elegant tool for modeling distributed applications not only on static distributed platforms but also in mobile distributed computing environments such as MANETs. In this section, we first lay the foundations of a task graph based modeling framework and then introduce the task embedding (or anycasting) problem. We then present an optimal polynomial-time algorithm for embedding a tree Task Graph onto a MANET, and then argue why the arbitrary graph embedding problem is hard. In Sec. 5 we present a more practical distributed algorithm which can function efficiently in dynamic, mobile environments.

\subsection{Definitions of Terms}

A device in our context is a physical entity that performs at least one particular function such as interaction with its physical surroundings, computation, and communication with other devices. It is usually equipped with an embedded processing element, sensors and actuators for interacting with the physical environment, a wireless communication port, and/or a user interface. If a device primarily performs one specific function, it is called a "specialized 
device", otherwise, it is referred to as a "multipurpose device". Examples of the former type include digital cameras, speakers, printers, keyboards, display devices etc., while examples of the latter include PDAs and laptops.

The capabilities of each device can be summarized in their attributes. Attributes can be static (which do not change with time) or dynamic (which change with time). For example, a network digital camera can have a static attribute "resolution" which can take values like 320x240, 640x480 etc. Examples of dynamic attributes include location (absolute or relative, depending on the availability of GPS), power levels, available computational power (or load), and available communication bandwidth. In this paper, we only consider specialized devices with their principal attribute, i.e., their main function. Multi-attribute extensions are possible to our work on the lines of [1].

A service is a functionality provided by a device or a collection of cooperating devices. Although hierarchical service composition ${ }^{3}$ is possible using our modeling scheme, in this work, we only consider services provided by single devices. Multiple devices can exist in the MANET for providing the same service.

A node is an abstract representation of a device or a collection of devices characterized by a minimal set of attributes that can offer a particular service. A node is simple when it represents a single physical device. It is complex when it represents multiple simple nodes. We consider simple nodes only in this paper. We refer to the principal attribute of a node or a device as its class or category or type. Examples of classes include printer, speaker, joystick etc.

An edge is a necessary association between two nodes with attributes that must be satisfied for the completion of a task. Examples of edge attributes include causal ordering, weight, required data rate between nodes, allowable bit error rate, and physical proximity.

\subsection{Tasks and Task Graphs}

A task can be described as work executed by a node with a certain expected outcome. The work done by a component of a complex node is considered a sub-task of the bigger task. An atomic task is an indivisible unit of work which is executed by a simple node. Atomicity is related to the core capability of a device (described through its attributes), and is partially

\footnotetext{
${ }^{3}$ creating more complex services from simpler services that are available in the current networked physical space.
} 


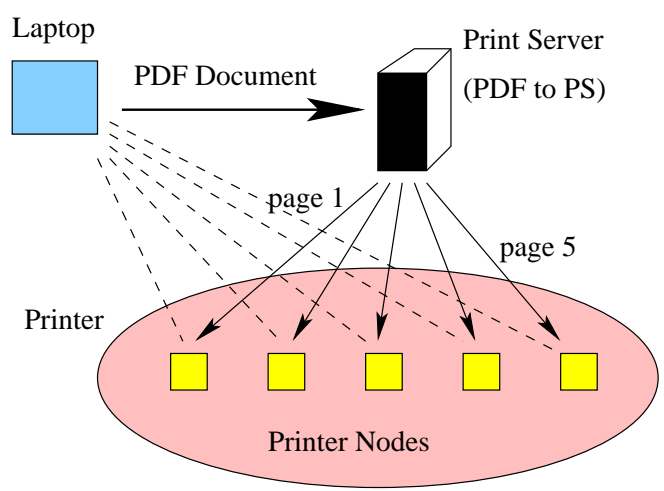

Figure 2: A Smart Printing Service

constrained by subjective design choices.

A task graph is a graph $T G=\left(V_{T}, E_{T}\right)$ where $V_{T}$ is the set of nodes that need to participate in the task, and $E_{T}$ is the set of edges denoting data flow between participating nodes.

An example may bring further clarity to the abstractions developed so far. Consider a scenario in which there is a postscript (PS) printer connected to a small computer (print server) running filtering software that can convert PDF files to printable PS format. The printer and the computer are each devices that offer a particular service. The printer is considered a specialized device offering the service of converting PS files into printed pages, while the computer is a multipurpose device which has among its many offered services the one service of converting PDF files into PS format. The example has been illustrated in Fig. 2 .

The printer is a physical device representation of a simple node with certain attributes (such as print resolution, ink color) that offer the service of converting PS files into printed pages. Analogously, the print server computer plus its filtering software can be viewed as a representation of a $P D F \rightarrow P S$ converter node. By taking these two nodes together we can form a complex node that offers a "PDF printing service". A task we have in mind is the printing of one PDF document. In this specific case, based on subjective criteria, we define an atomic task to be the printing of one page of the document ${ }^{4}$. The entire document can be then printed on a set of available printers as shown in Fig. 2. Note that in this scenario we formed a new service (PDF printing) by combining two existing ones. Granted that this

\footnotetext{
${ }^{4}$ We did not come up with any objectively quantifiable argument as to why the atomic task should not be the printing of a dot in the paper. Subjectively, we believe atomic operations at that level are too cumbersome to manage at such high-level abstraction.
} 


\begin{tabular}{|rl|rl|}
\hline (i) & $U:[-; S S][S S ;-]$ & (iv) & $M:[-; S S]$ \\
\hline \hline (ii) & $S S:[U ; L S, D][U ; U][K ; L S, D][M ; L S, D]$ & (v) & $L S:[S S ;-]$ \\
\hline \hline (iii) & $K:[-; S S]$ & (vi) & $D:[S S ;-]$ \\
\hline
\end{tabular}

Table 1: Tuples for the Presentation Task

example is simple (even trivial), we believe that research that enables such capability in today's MANETs for arbitrary device types and quantities is essential for tapping into the networked environment's full potential.

\subsection{A Data-flow Tuple Architecture}

Every class of device participating in a task possesses a set of tuples representing the task requirements in terms of the data flow from other classes of devices into the current class, and from the current class to other classes. Each tuple corresponds to a logical unit of data processing that is needed between the distributed components of an application. Consider the smart presentation application described in Sec. 3. The following data flows can characterize a sample presentation: (i) Presenter's PDA $(U)$ sends data to Smart Storage $(S S)$ with appropriate presentation software. $S S$ then displays the data on the Display $(D)$, Local Screen $(L S)$, and some corresponding notes on the PDA screen $(U)(\mathrm{ii})$ Keyboard $(K)$ data (from the presenter typing on the keyboard) and Mouse $(M)$ clicks are sent to $S S$ which interprets them and relays them to $D$ and $L S$.

To represent such application data flow between nodes we employ a generalized tuple architecture. If a node of class $X$ receives data from nodes of classes $A, B$ and $C$, and sends the processed data to nodes of classes $D$ and $E$ for a certain application flow (e.g. mouse commands or keystrokes), we can represent this flow using the following tuple: $X:[A, B, C ; D, E]$. Such an application data flow can be specified in a file using a standardized language and a Task Graph (TG) can be generated from it easily. The aforementioned

application data flows can be written as tuples as shown in Table 1 and they translate to the task graph shown in Fig. 1(a).

Having a tuple architecture for a task serves two purposes: (1) It is a structured specification of the data flows in a task from which a task graph can be derived, and (2) after the embedding of the task graph on the MANET, it governs the flow of actual application data at each participating device.

The example of a data-flow tuple presented above contains only the essential infor- 


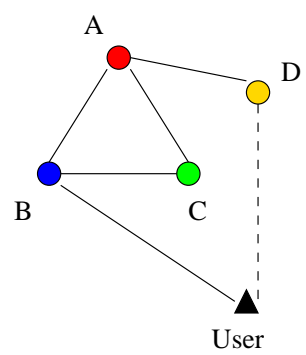

(a) Task Graph

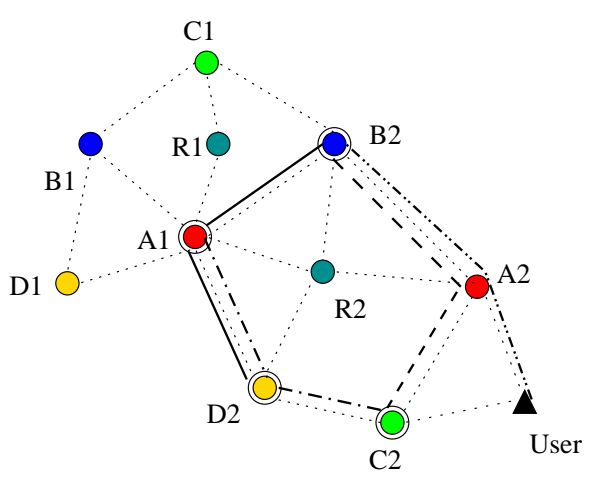

(b) Embedding \#1

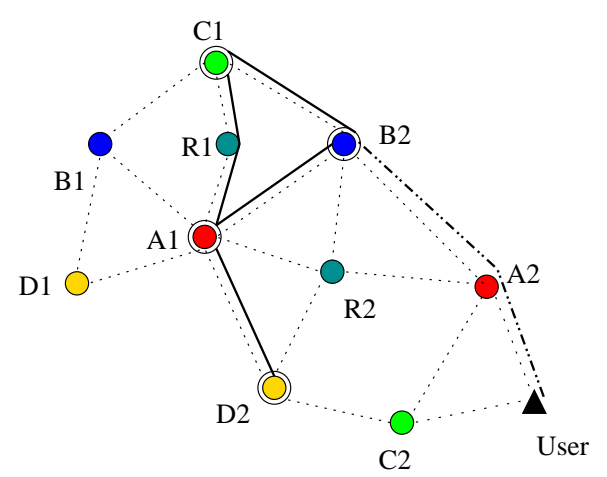

(c) Embedding \#2

Figure 3: Example of Task Graph Embedding

mation for data exchange, namely the data source and the data destination. In general, the edges in a TG can have attributes such as channel error rates, bandwidth, etc. which reflect the QoS needs of the distributed application. These, and requirements such as proximity (since devices like keyboard, mouse etc. should be located as near the user as possible) can also be integrated in the TG via the tuple architecture. One direct way of implementing it is to introduce a proximity attribute of the edge and specify it in the tuple. Implementation details of the proximity attribute are beyond the scope of this paper, and we reserve it for future study.

\subsection{Embedding Task Graphs onto Networks}

The first step in executing a distributed application on a set of specialized devices is to discover appropriate devices in the network and to select from those, some suitable devices that are needed to execute the more complex application. Mathematically speaking, embedding a task graph $T G=\left(V_{T}, E_{T}\right)$ onto a MANET $G=\left(V_{G}, E_{G}\right)$ involves finding a pair of mappings $(\varphi, \psi)$ such that $\varphi: V_{T} \rightarrow V_{G}$ and $\psi: E_{T} \rightarrow P_{G}$, where the class of $v \in V_{T}$ is the same as that of $\varphi(v)$ and $P_{G}$ is the set of all source-destination paths in G. Fig. 3(a) depicts a hypothetical task graph. Figures $3(\mathrm{~b}-\mathrm{c})$ show a sample network topology with two possible embeddings of TG on it.

The entire process of device discovery, selection of a device from multiple instances of devices in the same category, and the assignment of a physical device to a logical node in the task graph is referred to as instantiation. The collective process is called embedding or task-based anycasting. 


\subsection{Metrics for Performance Evaluation}

In general, the embedding process maps edges in $T G$ to paths in $G$. Average Dilation of an embedding is the average length of such paths taken over all edges in $T G$. Mathematically, if $\|a, b\|_{G}$ denotes the length of a shortest path between nodes $a$ and $b$ in $G$, dilation is given

by: $D_{\text {avg }}=\frac{1}{\left|E_{T}\right|} \sum_{(x, y) \in E_{T}}\|\varphi(x), \varphi(y)\|_{G}$. Dilation is an important metric since it impacts the throughput between instantiated devices. An embedding with large dilation signifies long paths between directly communicating devices, which is undesirable in MANETs since TCP throughput drops significantly with increase in hop distance [14]. In contrast, an embedding with low dilation results in better task throughput. We consider the weighted version of the metric in Sec. 4.6 where we formally describe the optimal embedding problem.

Instantiation time is another useful metric which measures the time taken to instantiate a node in $T G$ on $G$. When an embedding is disrupted owing to network failures, it also measures the time taken to find a replacement device.

A useful metric for measuring the resilience of the protocols to failures is Average Effective Throughput, (AvgEffT), which is the average number of application data units (ADUs) actually received at instantiated data sinks divided by the number of ADUs that were supposed to be received at the intended targets in an ideal situation ${ }^{5}$. Therefore, $0 \leq \operatorname{AvgEffT} \leq 1$

\subsection{Optimization Problem Formulation}

We formulated the minimum embedding problem as the following optimization problem:

Let $C$ be a set of principal attributes (or classes) of specialized devices. Let $G=\left(V_{G}, E_{G}\right)$ be a graph corresponding to a MANET of specialized devices, with the class of each device in $V_{G}$ belonging to $C$. Let $T G=\left(V_{T}, E_{T}\right)$ be a task graph such that the class of each node in $V_{T}$ belongs to some $S \subseteq C$. Also, a function $w: E_{T} \rightarrow \Re^{+}$defines edge weights which could signify data rate requirements or importance of edges with respect to the task. Find mappings $\varphi: V_{T} \rightarrow V_{G}$ and $\psi: E_{T} \rightarrow P_{G}$, where the class of $v \in V_{T}$ is same as that of $\varphi(v)$ and $P_{G}$ is the set of all "paths" in the network $G$, such that the weighted average dilation given

\footnotetext{
${ }^{5}$ If a relaying node in the path from source to sink gets uninstantiated, effective throughput will be affected since certain data flows will be dumped and will not reach the data sinks.
} 


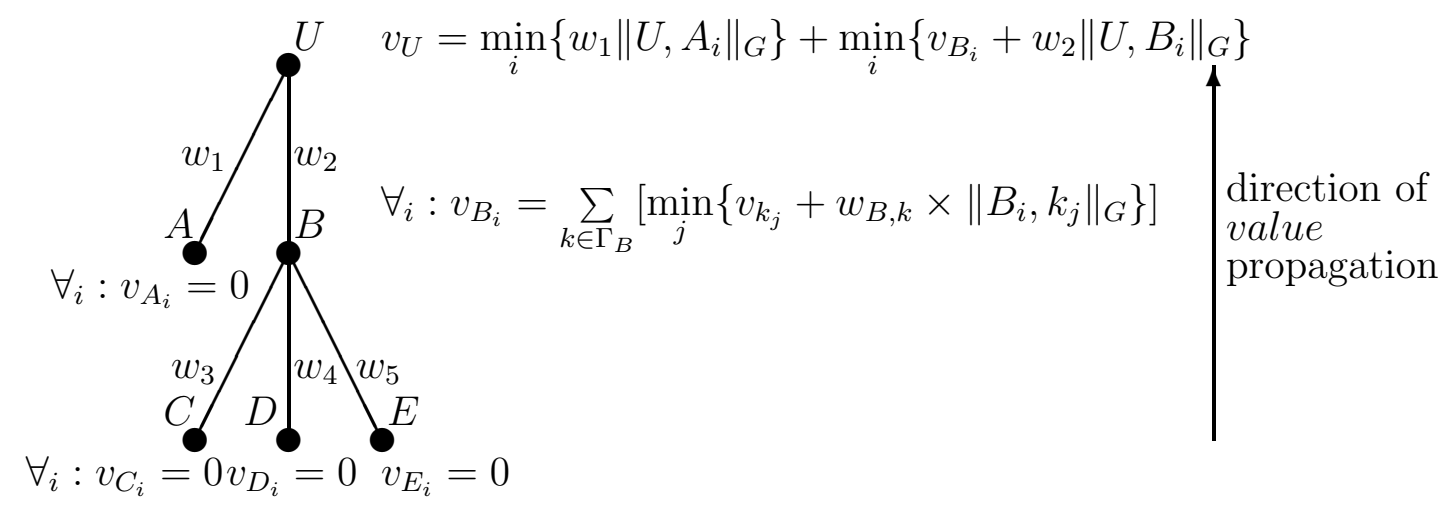

Figure 4: Optimal Solution based on the Principle of Optimality

by:

$$
D_{\text {avg }}=\frac{1}{\sum_{e \in E_{T}} w(e)} \sum_{e=(x, y) \in E_{T}} w(e)\|\varphi(x), \varphi(y)\|_{G}
$$

is minimized, where $\|a, b\|_{G}$ denotes the shortest path between devices $a$ and $b$ in $G$.

\subsection{An Optimal Polynomial-time Algorithm for Embedding Tree Task Graphs}

We present an optimal algorithm (with respect to $D_{\text {avg }}$ ) for embedding or anycasting a tree task graph onto a MANET at a given instant of time. The running time is polynomial in $|G|$ as well as $|T G|$. The algorithm minimizes searching in the solution space by taking advantage of the tree structure of TG, and is based on the principle of optimality ${ }^{6}$. The algorithm requires that the node executing the algorithm have a complete knowledge of the topology of the network at the given instant of time.

The algorithm proceeds by the propagation of a certain value function from the leaf nodes of $T G$ towards the root node $U$. It comprises of the following steps:

1. Perform a BFS traversal of the tree $T G$ and assign a level $L$ to each node starting from $U$ which has $L=0$.

\footnotetext{
${ }^{6}$ Principle of Optimality holds for problems whose structure is such that their optimal solutions contain the same for the smaller sub-problems.
} 
2. Now begin assigning a value, $v$ to nodes starting from the highest level, $L_{\max } \leq\left|V_{T}\right|$. If the node under consideration, say $X$, is a leaf node in $T G$, for all instances $X_{i}$ in $G$ of class $X$, assign value zero, i.e., $\forall_{i}: v_{X_{i}}=0$.

3. If $X$ is not a leaf node, consider its children at every instance $X_{i}$ of $X$ in $G$. For instance $X_{i}$, for every child node $Y$ of $X$, carry out the following optimization step: sweep through all the instances $Y$ in $G$, and select the instance $Y_{j}$ which minimizes the quantity $v_{Y_{j}}+w_{(X, Y)}\left\|X_{i}, Y_{j}\right\|_{G}$.

The crux of the idea is that the principle of optimality holds because of the tree structure of $T G$ : if $X_{i}$ is selected by its parent and is optimal, then the choice of $Y_{j}$ is optimal too. This greatly reduces the search space. After carrying out this step for all children of $X$ for $X_{i}$, assign the sum of the calculated minimum values to $v_{X_{i}}$. Fig. 4 illustrates the procedure for a task graph of 6 nodes $^{7}$.

4. Complete this value assignment for all instances of all nodes at a given level in $T G$ before considering lower level nodes. At $U$, choose the children such that $v_{U}$ is minimized (note that there is only one instance of $U$ ). Now, we have the optimal instances of all nodes which minimize the dilation metric in Eq. 1, and hence have an optimal embedding.

Now, we calculate the running time of the above algorithm. Step 1 takes $O\left(\left|V_{T}\right|+\left|E_{T}\right|\right)$ time. If the nodes with the same level are pushed onto a stack after traversal, then nodes with $L=L_{\max }$ will reside at the top and value propagation can occur efficiently. Now, since in the worst case, $L_{\max }=\left|V_{T}\right|=O\left(\left|V_{T}\right|\right.$ ) (although in more balanced trees, it is logarithmic in $\left.\left|V_{T}\right|\right)$, Step 2 can loop $O\left(\left|V_{T}\right|\right)$ times. In Step 3, every edge in $T G$ is mapped to a shortest path in $G$. Suppose there are $|C|$ classes of devices in $G$ with $\frac{\left|V_{G}\right|}{|C|}$ instances of each class. For every parent instance, each child instance is considered, the shortest path is computed (in $O\left(\left|V_{G}\right|^{2}\right)$ time) and a minimization step performed (in $O\left(\frac{\left|V_{G}\right|}{|C|}\right)$ time). Hence this mapping process for all edges will take $O\left(\left|E_{T}\right| \times \frac{\left|V_{G}\right|}{|C|}\left(\frac{\left|V_{G}\right|}{|C|} \times\left|V_{G}\right|^{2}+\frac{\left|V_{G}\right|}{|C|}\right)\right)=O\left(\left|E_{T}\right| \frac{\left|V_{G}\right|^{4}}{|C|^{2}}\right)$ time. Note that the Step 2 loop is subsumed in this calculation. Since $\left|V_{G}\right|$ is the dominant term, the time complexity is given by the above expression itself. If we use All Pairs Shortest Path algorithm (running time is $O\left(\left|V_{G}\right|^{3}\right)$ and extraction of shortest path cost is $O(1)$ assuming a random access storage) instead of the Dijkstra's Source-Destination Shortest Path, then running time of the entire process is $O\left(\left|E_{T}\right| \frac{\left|V_{G}\right|^{2}}{|C|^{2}}+\left|V_{G}\right|^{3}\right)=O\left(\left|V_{G}\right|^{3}\right)$.

\footnotetext{
${ }^{7} \Gamma_{B}$ is the set of children of $B$ in TG. $k_{j}$ is an instance in $G$ of child $k$ of $B$ in TG.
} 
This algorithm has the following drawbacks: (1) it is centralized and does not have a low time complexity, (2) it needs entire topology information at a particular device which we believe is unrealistic for large MANETs that we consider in this paper, and (3) it is not adaptive to mobility of devices. Hence, in Section 5 we propose a distributed greedy heuristic approach which albeit suboptimal, is much simpler and less time consuming, and reasonably efficient in operation.

Now, if $T G$ is a general graph (and not a tree), then the task embedding problem becomes much harder. The principle of optimality may not hold in case of general graphs because the optimal embedding of every pair of nodes and the edge connecting them cannot be done independent of other edges and nodes in $T G$, as can be done if $T G$ were a tree. In case of a tree, as we propagate the values from the leaves to the root, the optimal embeddings of each sub-tree are retained and used later while embedding a node with lower value of $L$. This is not possible for any general task graph with greater connectivity than a tree.

Since there are $\left|V_{T}\right|$ distinct classes of devices in $T G$, and $\frac{\left|V_{G}\right|}{|C|}$ instances of each class in the network $G\left(\left|V_{T}\right| \leq|C|\right)$, the total number of possible embeddings is $\frac{\left|V_{G}\right|^{\left|V_{T}\right|}}{|C|^{\mid V_{T}}}$. Hence, the search space for an optimal brute force algorithm is exponential in $\left|V_{T}\right|$, which is clearly unacceptable. The combinatorial structure of the problem prompted us to look for a heuristic solution, the basic idea of which is very simple: find a spanning tree $S T_{\max }$ of $T G$ with the maximum weight and then embed that tree in $G$ using the algorithm described before. It is easy to see that the edges of $T G$ which are not in $S T_{\max }$ will not be considered by the embedding process and can be mapped to very long paths and give highly suboptimal results without any bounded approximation factor. Any better solution will have to involve partitioning $T G$ into smaller components which can be embedded independent of each other. Finding such components and then embedding them onto the network both are likely to have high time complexity. Hence, we trade off complexity for optimality and look for greedy heuristic solutions which are simple and distributed in nature.

\section{A Distributed Task Embedding Approach}

A centralized approach is often preferable when there is some existing static infrastructure with a fixed base station node or a directory server, devices are less mobile and more connected with each other, and the probability of node and link failure is low. In the Sec. 4.7 we described a centralized solution which requires knowledge of the entire network and yields optimal results for tree TGs. However, when we consider a dynamic and mobile distributed 

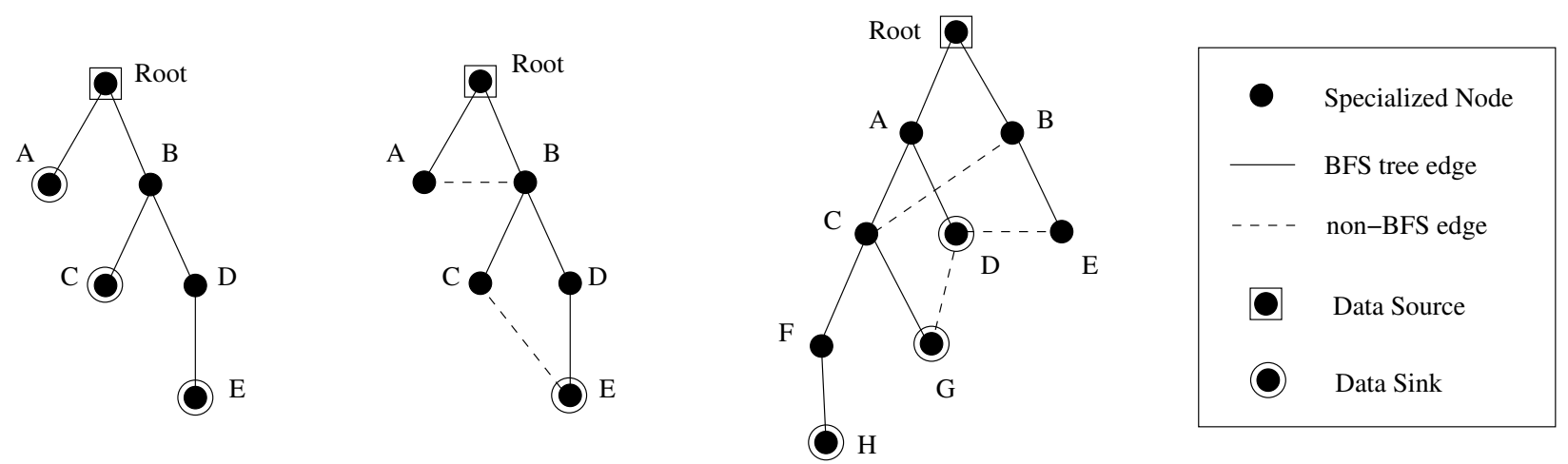

Figure 5: Task Graphs: (a) Tree, (b,c) Non-Tree Graphs

environment such as a MANET of low power devices, no device in the network may possess adequate computational power to execute that algorithm. Also the MANET graph could change by the time the optimal dilation is calculated. Moreover, it is hard for one device to track the changes in topology between participating instances using reactive MANET routing protocols such as DSR.

It is also hard to guarantee that a device acting as a centralized controller will always be connected to the rest of the network. When a disconnection or partition occurs in the network, a new controller may have to be elected, and that device will have to compute a new embedding. Although it may be easier to find close-to-optimal solutions (with respect to particular performance metrics) using a centralized approach than a distributed one, the latter is more robust and adaptive to mobility since there is no single point of failure. Hence, we feel that localized distributed algorithms are better suited for such dynamic MANET environments.

Services such as device discovery and selection can be implemented in an intermediate layer (say, TG layer) between the application and network layers. Our approach assumes the presence of routing support in the MANET. However, one can also envision the TG layer to be embedded in the network layer for performance improvements (not considered in this work).

\subsection{A Distributed Algorithm for Instantiation of Nodes}

In this section we present a distributed algorithm for the instantiation of nodes (or anycasting of the corresponding service) in a given task graph. The corresponding distributed protocol has been specified by the finite state machines shown in Figs. 7 and 8 . 
The principal goal of the algorithm is to produce an embedding of a TG onto a MANET with the objective of minimizing $D_{\text {avg }}$. In this work, we assume that each heterogeneous device can provide a single type of service, and that all nodes in the network are simple. Our concept can be easily extended to incorporate the case where multiple types of services are provided by one particular device. We assume the presence of a MANET routing protocol (e.g. DSR, AODV etc.) and a reliable transport protocol (TCP).

All devices in the network execute copies of the same algorithm except the user node, $U$ which executes a different algorithm since it acts as a state synchronizer (or coordinator) in the initial phases of the embedding process. Any device in the network other than the main coordinator can exist in one of the following states at any instant of time: NOT_INSTANTIATED, WAIT_FOR_ACK, or INSTANTIATED. The main coordinator can exist in TG_UNINSTANTIATED or TG_INSTANTIATED state. A task graph TG is submitted by the user node's application layer to its TG layer which begins the embedding process.

Initially the coordinator $U$ is in state TG_UNINSTANTIATED (which means the task graph has not been instantiated yet) and all other nodes are in state NOT_INSTANTIATED (the node has not been instantiated by the coordinator). The embedding process begins at $U$ with a distributed search which proceeds through the MANET $G$ hand-in-hand with a breadth-first search (BFS) through TG. Fig. 5(c) depicts a task graph with its BFS and non-BFS edges. We call the spanning tree on $T G$ induced by BFS and rooted at $U$, a BFStree $\left(B F S T_{T G}\right)$ of $T G^{8}$. We propose a greedy solution to keep the dilation of the embedding low: the algorithm begins from $U$ by progressively mapping the nodes of $B F S T_{T G}$ to nearest devices and the edges to shortest paths in $G$. Instantiation of any pair of nodes $x, y \in V_{T}$ cannot affect each other if $x$ is not a parent of $y$ in $B F S T_{T G}$, or vice versa. Hence, the search can proceed in a distributed manner along the branches of $B F S T_{T G}$. Note that unlike the optimal algorithm proposed in Sec. 4.7, this algorithm greedily instantiates nodes in $T G$ starting from $U$ and searches only the local space around an instantiated device for instantiating the next node.

The salient steps of the algorithm have been illustrated in Fig. 6 by means of a time-based message diagram. First, nodes which are neighbors of the user node, $U$ in TG are visited parallely, and their instances are discovered in the network. $U$ issues broadcast

\footnotetext{
${ }^{8} \mathrm{We}$ assume here that all edges of $T G$ have equal weight. If the weights are unequal, a Maximum Weight Spanning Tree should be computed and its edges should be used for performing the embedding instead of the BFS-tree. Rest of the steps are similar to the ones taken for embedding a BFS-tree with obvious changes in nomenclature.
} 


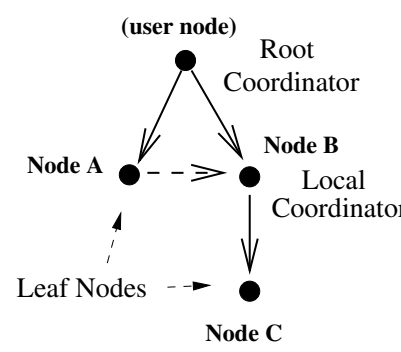

TASK GRAPH

$\longrightarrow$ BFS-tree edges

$-\rightarrow$ Non-BFS-tree edges

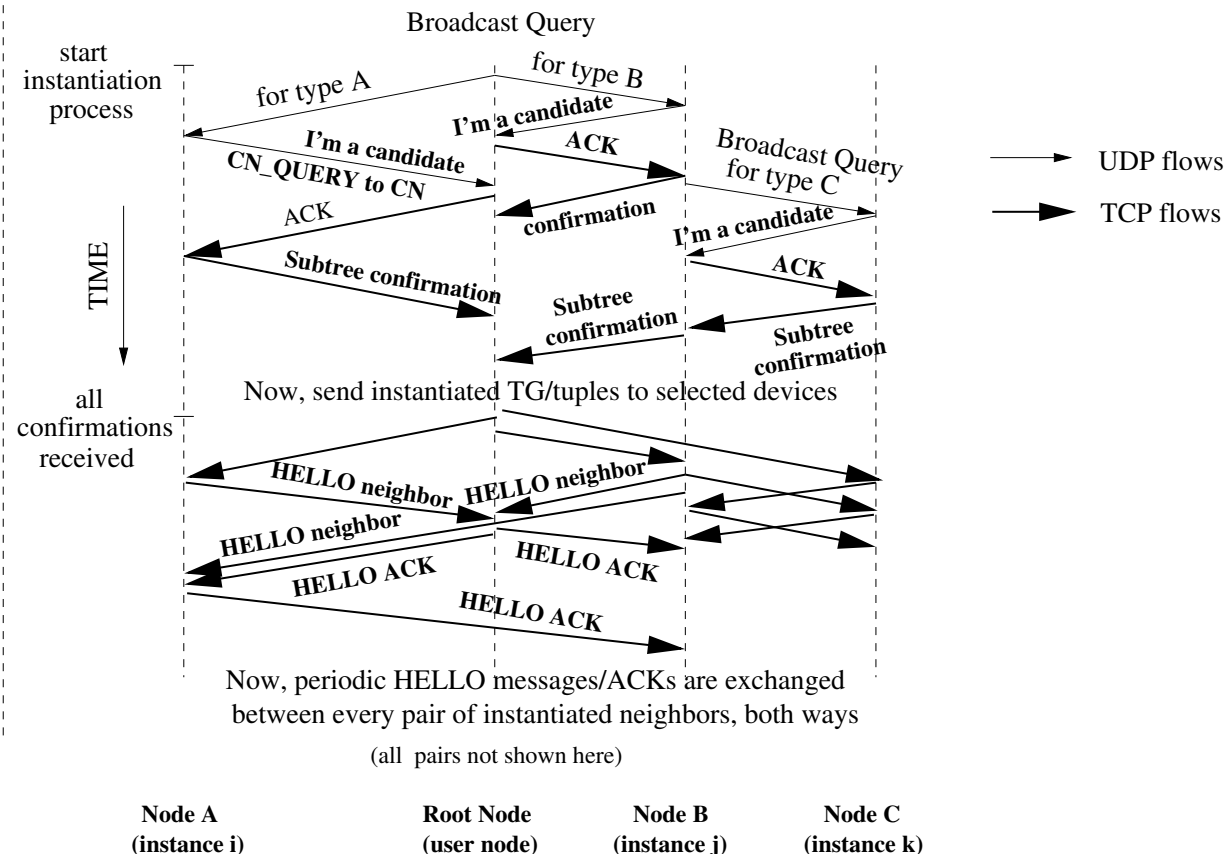

Figure 6: Dynamics of the Embedding Scheme

search queries for each neighbor (types $\mathrm{A}$ and $\mathrm{B}$ ) in $\mathrm{TG}^{9}$. The query types in the search packet are A and B respectively. A packet is rebroadcasted by the TG layer of a device, $D$ if it does not match the search query type, the time-to-live (TTL) value in the packet has not reached zero, and if $D$ has not seen the same broadcast (identified by broadcast sequence numbers) before.

When a free instance $B_{j}$ of type $\mathrm{B}$ gets a search packet, it sends a candidate query packet to the synchronizer/coordinator $U$ expressing its willingness to participate in the task. $B_{j}$ changes its state to WAIT_FOR_ACK. $U$ checks the state of the instantiation of TG node B and sends an acknowledgment to $B_{j}$ if $\mathrm{B}$ has not been instantiated yet in its TG. The coordinator $U$ is responsible for accepting and rejecting replies from several candidate devices in the network before the discovery is completed. The first candidate device that replies to a search query packet of type B becomes the instance of $\mathrm{B}$ at $U$.

Along with the acknowledgment, $U$ also sends a list of device types that are in the sub-tree of TG rooted at B (such as C). $U$ does not send explicit negative acknowledgments to all other instances of $\mathrm{B}$. Instead, a candidate $B_{k}$ starts a timer as soon as it sends a candidate query to $U$. If the timer expires before it receives an ACK, it puts itself in

\footnotetext{
${ }^{9}$ The broadcast is controlled by executing it at the TG layer rather than the IP layer. Essentially, a device in the network broadcasts a packet to all its one-hop neighbors which examine the state of the packet and decide whether to rebroadcast it or not.
} 
NOT_INSTANTIATED state again. This is more scalable than $U$ sending NACKs, albeit at the cost of tying $B_{k}$ in WAIT_FOR_ACK state for a longer time. An acknowledged candidate $B_{j}$ changes its state to INSTANTIATED, and confirms its role to $U$. If there are any uninstantiated nodes rooted at $\mathrm{B}$ in the $\mathrm{TG}$, then $B_{j}$ issues broadcast search query packets for all those node types and the instantiation proceeds further. $B_{j}$ acts as a local coordinator for all nodes in its sub-tree. After receiving individual confirmations from selected node instances in its sub-tree, it aggregates them into one collective confirmation packet and sends it to $U$. When $U$ receives confirmations for all node types in TG, it sends the instantiated-TG and tuples to the selected devices.

The details of our protocol have been illustrated in Figs. 7-8 by means of finite state machines (FSM diagrams) that need to run on the devices in the network for finding the embedding. In our opinion, the user devices are best suited for acting as coordinators since they usually originate the application data flows, and even under mobility, always remain near the user.

After the instantiation information is exchanged, the user application can start data transmission. The flow of data will be governed by the instances of the corresponding tuples, and in the ideal situation, all data originating at the source should reach the instances of the sink nodes in TG $\left(A_{i}\right.$ and $C_{k}$ in the example in Fig. 6) after being relayed by the intermediate devices $\left(B_{j}\right)$.

The task graph itself is sent as control data during the instantiation process. Once the instantiation process is complete, the selected set of devices communicate with each other using the underlying TCP layer which sits on top of the MANET routing layer (DSR in our case). TCP is used to exchange control and data packets since packet losses due to route errors are very common in MANETs. Our protocol does not assume anything specific about the routing protocol running on the MANET although we favor reactive/ondemand protocols in principle over proactive ones. Hence we chose DSR for our simulation experiments.

\subsection{Handling Mobility of Devices}

In this section, we present in detail how our instantiation algorithms react to the mobility of devices. If the devices in the network are highly mobile during the lifetime of a distributed application running on the network, the network topology and previously established con-

nections may change, and this may disrupt the application. Therefore, in the presence of 


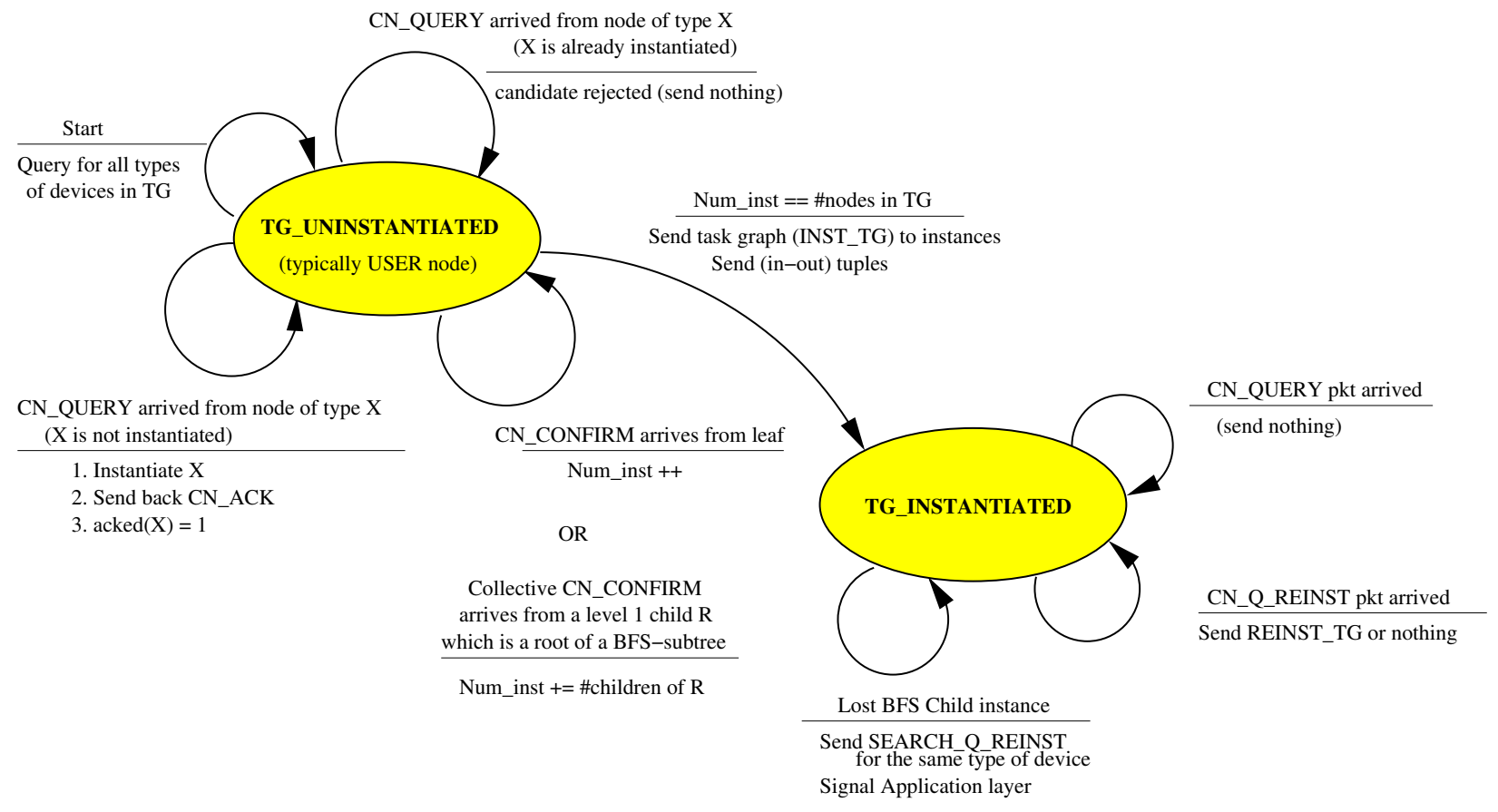

Figure 7: Coordinator Device Protocol FSM

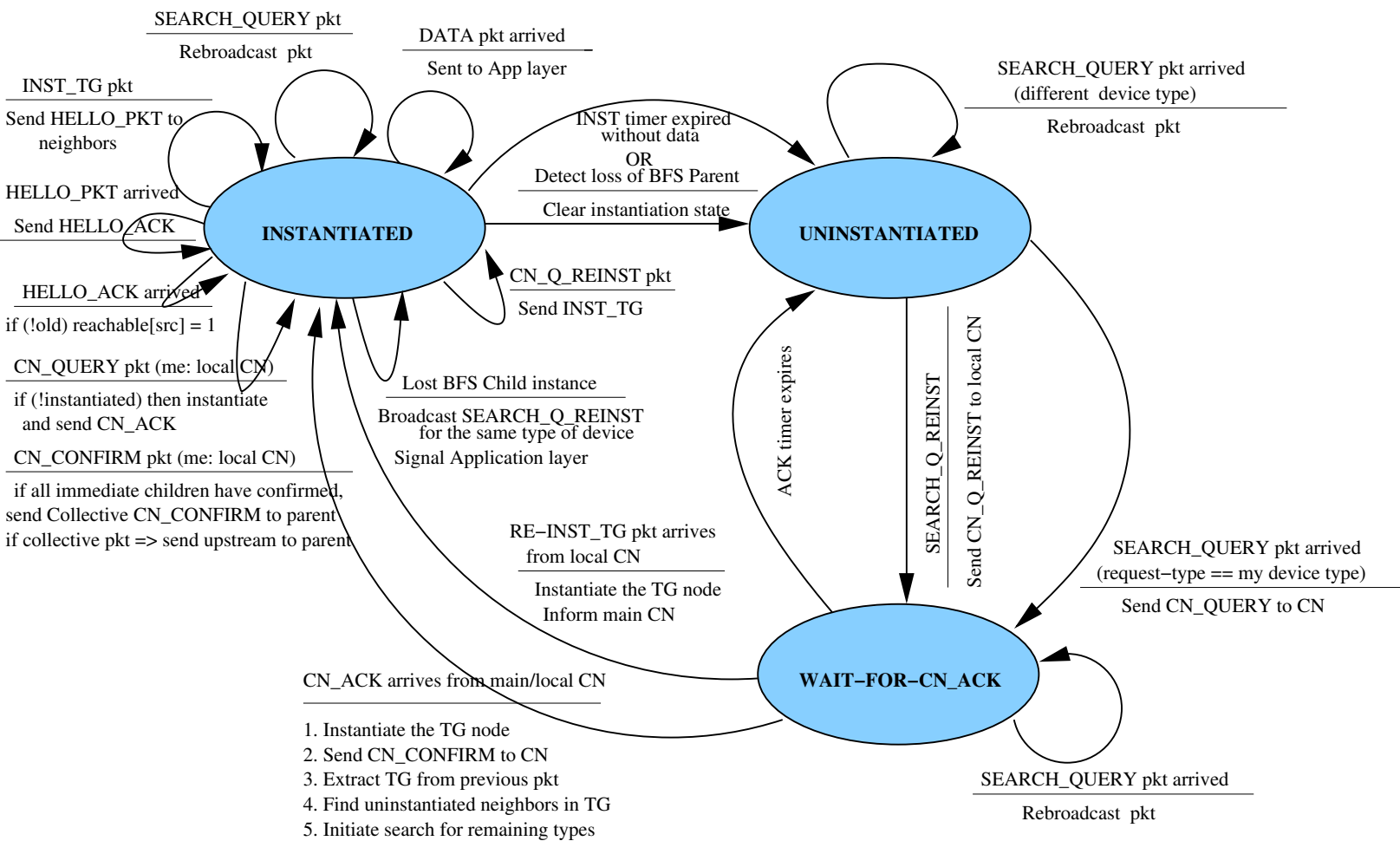

Figure 8: Non-Coordinator Device Protocol FSM 

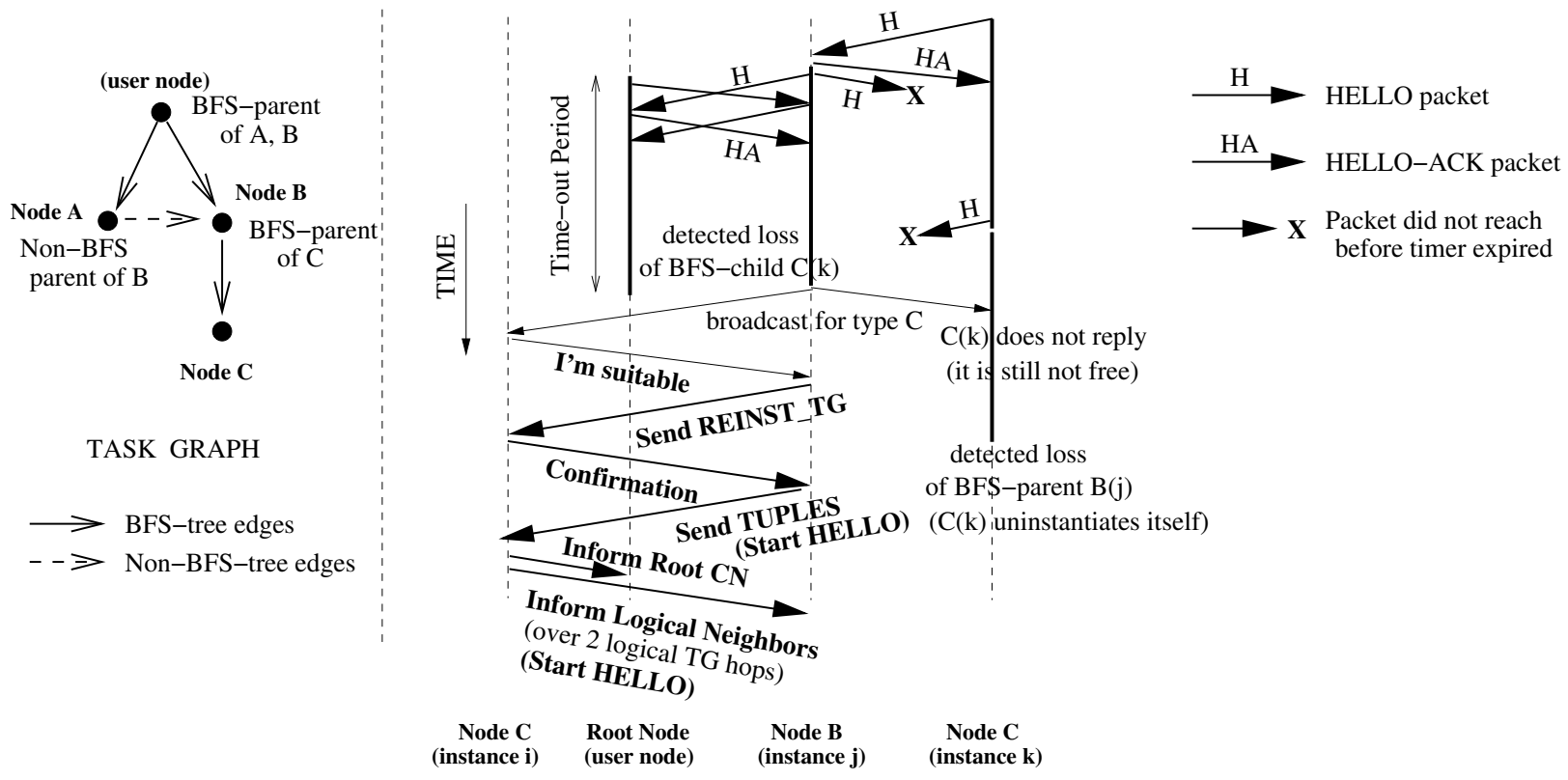

Figure 9: Re-instantiation of Devices

mobility, it is no longer sufficient to permanently select specific devices and appoint them to execute the application - continual monitoring must be performed for detecting disruptions, and replacement devices must be selected for resuming the application.

\subsubsection{Detection of Disconnections}

When mobility causes network partitions or disconnections, the instantiated devices may no longer be able to communicate if the partition breaks all paths between them. In such situations, new instances need to be selected. The necessary first step in this direction is the detection of disconnections.

Mobility of devices may cause network partitions or disconnections, and two instantiated devices may no longer be able to communicate if all paths between them are broken. We propose a lightweight, soft-state exchange protocol for detecting disconnections in an instantiated TG. The protocol requires each instantiated device to send periodic HELLO messages (with period $T$ ) to its logical neighbor instances in TG, which reply with a HELLO-ACK. This has been demonstrated in Fig. 9.

Each instantiated device specially keeps track of its BFS parent and BFS children. For example, in Fig. 5(c), the instance of type $\mathrm{C}$ keeps track of the instance of type A (its BFS parent) as well as of the instances of types $\mathrm{F}$ and $\mathrm{G}$ (BFS children). If a BFS 
parent device stops hearing from one of its BFS children ${ }^{10}$, it uninstantiates its child and starts searching for a replacement of the same type. The child meanwhile would stop hearing HELLO-ACKs from the parent (assuming bidirectional links), and will uninstantiate itself. This has been illustrated in Fig. 9. On average, if the HELLO timer is set to $T$ seconds at every instantiated device, disconnections will be detected after $\frac{T}{2}$ seconds. State transitions that result from this process have been shown in the FSM diagrams in Figs. 7 and 8.

Mobility of devices may also result in lengthening or shortening of routes between device instances, and ideally, if there is no disconnection/partition, the application should proceed without disruption. But such ideal conditions may not hold in reality where route failures can trigger route discovery which along with TCP re-transmissions after timeouts may sometimes take several seconds to complete. Hence, this can result in HELLO-ACKs not coming back in $T$ seconds which results in the conclusion that a disconnection has happened, even when the nodes are reachable from one another.

Recently, researchers have proposed solutions to the above problem based on explicit notification of route errors to TCP [8]. In this study, we do not attempt to alter TCP or DSR (including their default timer settings), and simply build our protocol on top of these protocols. Hence, if a device does not receive a HELLO-ACK from its neighbor in $T$ seconds, we deem the neighbor to be disconnected. A reasonable value of $T$ is one which is not low enough to cause significant control overhead ${ }^{11}$, and not high enough such that disconnections are not detected fast enough. For our simulations, we chose $T=7$ seconds $(>6 s$, the default TCP re-transmission timer).

\subsubsection{Process of Re-instantiation and Bookkeeping}

If a BFS parent device detects a disconnection with its child at the expiry of its HELLO timer, it issues a broadcast search query for the same type of the child. The re-instantiation process then proceeds like the earlier instantiation process with a small simplification that the CN_CONFIRM step is omitted, since the BFS parent here handles one disconnection at a time. Also, the coordinator device is not involved in this process, and the BFS parent device acts like a local coordinator. After finding a new device, the BFS parent instantiates it in its own copy of TG, and sends a portion of the re-instantiated TG to the new child device.

\footnotetext{
${ }^{10}$ The parent concludes this if it does not get a HELLO-ACK from that child before the expiry of its HELLO timer

${ }^{11}$ Although exchanging HELLO messages with higher frequency could result in the DSR caches having fresher routes
} 


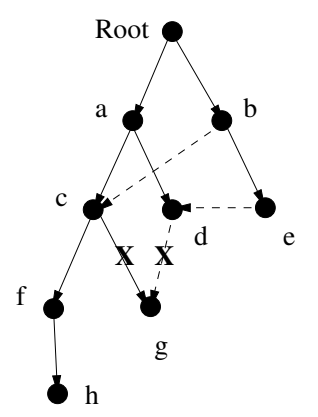

g gets disconnected from c c detects disconnection c searches for new $\mathrm{g}$ d does not do anything

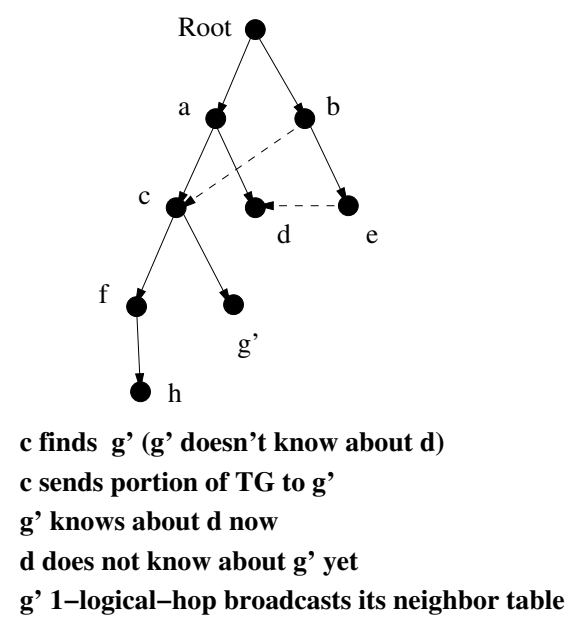

d knows about g' now c knows that g' knows about d c,d 1-logical-hop broadcast their neighbor tables a,b,e come to know about $\mathrm{g}$ ' (this is useful if $\mathbf{c}$ or $\mathbf{d}$ are disconnected)

Figure 10: Bookkeeping after Re-instantiation

Fig. 10 helps appreciate the reason behind doing this. $a, b, \ldots, h, g^{\prime}$ are device instances.

Our protocol addresses the problem of state maintenance after disconnections, and attempts to solve it locally, i.e. without involving the coordinator node. Each instantiated device knows the addresses of its parents (both BFS and non-BFS), its children, its children's parents, and its children's children ${ }^{12}$. With a little thought, one can see that information about this portion of TG is enough to handle single node disruptions in the instantiated task graph. However, there is one situation which cannot be adequately handled by this technique: a network partition such that 2 nodes connected by a non-BFS edge get disconnected from their parents. e.g., $d$ and $g$ get disconnected from $c$ and $a$ respectively, in the same HELLO period. $a$ finds $d^{\prime}$, and $c$ finds $g^{\prime}$ but $a$ tells $d^{\prime}$ about $g$ and $c$ tells $g^{\prime}$ about $d$ (from their 2-logical-hop neighbor tables), and a deadlock situation occurs where $d^{\prime}$ tried to contact $g$ and $d^{\prime}$ tries to contact $d$. To solve this, we adopt an approach where a non-BFS parent asks for the coordinator's help if it loses its non-BFS child. Since the coordinator is kept informed by newly instantiated nodes, such a situation can be avoided by taking its help. Note that if the coordinator is unreachable from this node instance, it is because of a network partition alone (since we do not have any timers for such messages), and in that case, a chunk of the instantiated network has been disconnected from the rest (containing the coordinator). In such situations, repair occurs soon at the BFS-parent node instances whose HELLO timers expire.

\footnotetext{
${ }^{12}$ Essentially, this is 2-logical-hop information except for parent's parents information which is unnecessary.
} 


\subsubsection{Impact of Disruptions on the Application Layer}

The application layer of every participating device keeps up-to-date (in-out) tuple information for parent and children devices. If the running task gets disrupted by disconnection of some participating devices, then it is the responsibility of the BFS-parent device to transfer the application state to the newly instantiated replacement device, and then resume the application data-flow. Meanwhile in data packets reaching old device instances are dropped by those devices which would be in the NOT_INSTANTIATED state after being disconnected. Our average effective throughput metric tries to capture the effectiveness of our disruption handling algorithm by measuring the fraction of the data that actually made it to the data sinks from the source. A higher layer buffer management scheme at the BFS-parent device instance can increase the reliability of task completion.

\begin{tabular}{|l|l|}
\hline Simulation Parameter & Value \\
\hline \hline Number of Devices & 50,100 \\
\hline Simulation Area & $1000 \mathrm{~m} \times 1000 \mathrm{~m}$ \\
\hline Transmission Radius & $250 \mathrm{~m}$ \\
\hline Mobility Model & Random Waypoint $([7])$ \\
\hline MANET Routing & DSR \\
\hline \#Classes of Devices & $12^{\S}$ \\
\hline Size of TG: $\left(\left|V_{T}\right|,\left|E_{T}\right|\right)$ & $(6,5),(6,7),(9,11)$ \\
\hline Simulation Time & $600 \mathrm{~s}$ \\
\hline MaxSpeed & $1-20 \mathrm{~m} / \mathrm{s}$ \\
\hline Pause Time & $0-600 \mathrm{~s}$ \\
\hline $\begin{array}{l}\text { Data Rate at Source } U \\
(B=\text { Burst size }(\text { bytes }),\end{array}$ & $(12500,5):$ medium \\
$P=$ Time period $(\mathrm{sec}))$ & $(1000,10):$ low \\
$(2500,1):$ freq. medium \\
\hline
\end{tabular}

Table 2: Simulation Parameters, $\mathrm{Tx}=250 \mathrm{~m}$

\section{Simulation Results}

We simulated the algorithms (and protocols) described in Sec. 5 using the popular network simulator $n s-2[22]$ for two different scenarios. The simulation parameters for the scenario depicting a large open area with nominal radio transmission range of $250 \mathrm{~m}$ have been listed in Table 2. We show simulation results for task-graphs in Fig. 5 - we refer to them as Tree TG, Non-Tree TG-1 and Non-Tree TG-2 respectively. The instantiation process began at

\footnotetext{
${ }^{\S}$ With uniform probability distribution
} 


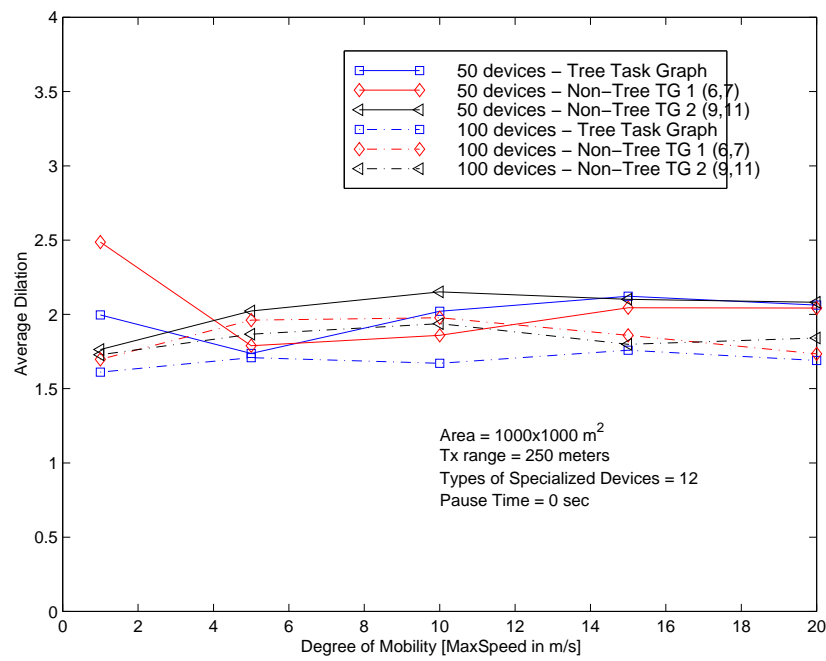

Figure 11: Average Dilation vs. Speed

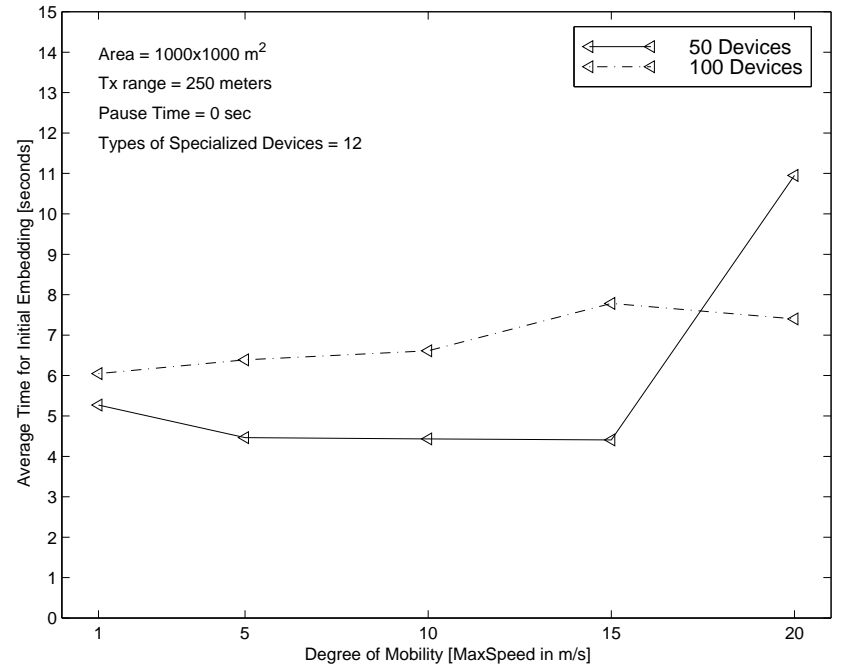

Figure 12: Average Time for Embedding of TG-2

$50 \mathrm{~s}$, and at $60 \mathrm{~s}$, a CBR source at the user/root node started sending data to the data sinks at the three different load granularities specified in Table 2. The results have been averaged over three different random specialized device patterns on two different sets of mobility scenario files. Devices which are not part of the current instantiated TG do not relay data packets, and such packets are not buffered ${ }^{13}$.

We begin with results for the medium load scenario: $(B, P)=(12500,5)$. We consider the constant mobility scenario (PauseTime $=0$ ) for a range of maximum speeds. We observe from Fig. 11 that the average dilation for the MANET with 100 devices does not vary greatly with speed. This means that the average number of physical hops between two instantiated nodes in TG is low and remains approximately constant under mobility. Actually, even if a HELLO message does not reach a device within expiration time, owing to the uniform distribution of device categories in space, the re-instantiation process will find another device with similar attributes within a range that keeps the dilation value constant. A similar pattern is observed for the network with 50 devices, although $D_{\text {avg }}$ is higher in that case owing to a $50 \%$ lower device density.

Fig. 12 compares the average time taken for embedding TG-2 on both MANETs. We observe that in general, more time is taken for embedding/anycasting the same TG on the denser MANET although devices are likely to be found closer on average in that MANET (as is observed from the dilation curves). This can be explained by the fact that

\footnotetext{
${ }^{13}$ In other words, if a device which was part of a TG becomes disconnected while there is a packet in transit, the packet is lost.
} 
SEARCH_QUERY broadcast traffic occupies significant bandwidth in the initial phase of the embedding process (even after controlling it by mechanisms mentioned in Sec. 5.1), thus delaying the flow of control packets such as CN_ACK and CN_CONFIRM. Also, there are more instances of each category which receive SEARCH_QUERY broadcasts in the denser MANET and a co-ordinator device takes more time to process their responses while proceeding with the instantiation process. Hence the aggregate embedding time increases.

We plot AvgEffT for different TGs for both 50 and 100 device MANETs in Fig. 13 (top two figures). We can see clearly that in general, effective throughput drops with increase in mobility speed. In the figures at the bottom of Fig. 13, we plot the average number of re-instantiations underwent during the entire simulation time. The rate of change in network topology increases with speed causing more network partitions (mainly in the 50 device MANET) or route errors (in both MANETs). These in turn prevent HELLO packets from arriving in time, and this triggers more re-instantiations. Since packets caught in transit during the re-instantiation process are dropped (as mentioned earlier, we do not consider application layer buffering in this work), AvgEffT is directly affected by re-instantiations.

In spite of Tree TG having more re-instantiations than TG-1 (we will explain the reason for this phenomenon in the next paragraph), it yields better AvgEffT. This is because the data tuples of TG-1 (as well as TG-2) involve flows along non-BFS edges in the graph. Also, here we are only plotting the re-instantiations, and not all disruptions. When a non-BFS parent loses a child instance momentarily due to HELLO timeouts, a reinstantiation will not be triggered since that is the responsibility of the BFS parent of the child instance; Hence, the throughput is affected until a new instance is found by a BFS parent and the non-BFS parent is informed of this event by a 1-logical-hop broadcast (as shown in Fig. 10), or a route to the old instance is restored. Also, Tree TG has sinks at all depths unlike TG-1 - the latter's effective throughput suffers more from a re-instantiation of an intermediate relay node.

Although Tree TG is a sub-graph of TG-1, it suffers more re-instantiations because data flow along the non-BFS edges og TG-1 results in the presence of more valid alternate routes (or parts of them). Hence, when a route error happens along a BFS edge (the primary cause of re-instantiations) of TG-1, often these alternate routes come to the rescue before the HELLO timer expires, thus reducing the rate of re-instantiations. TG-2 generally suffers more re-instantiations since it is a larger graph with more depth.

We can observe that TG-1 and TG-2 generally have much lower throughput than TreeTG for the dense MANET case, especially at moderate to high speeds. Most of this drop in 

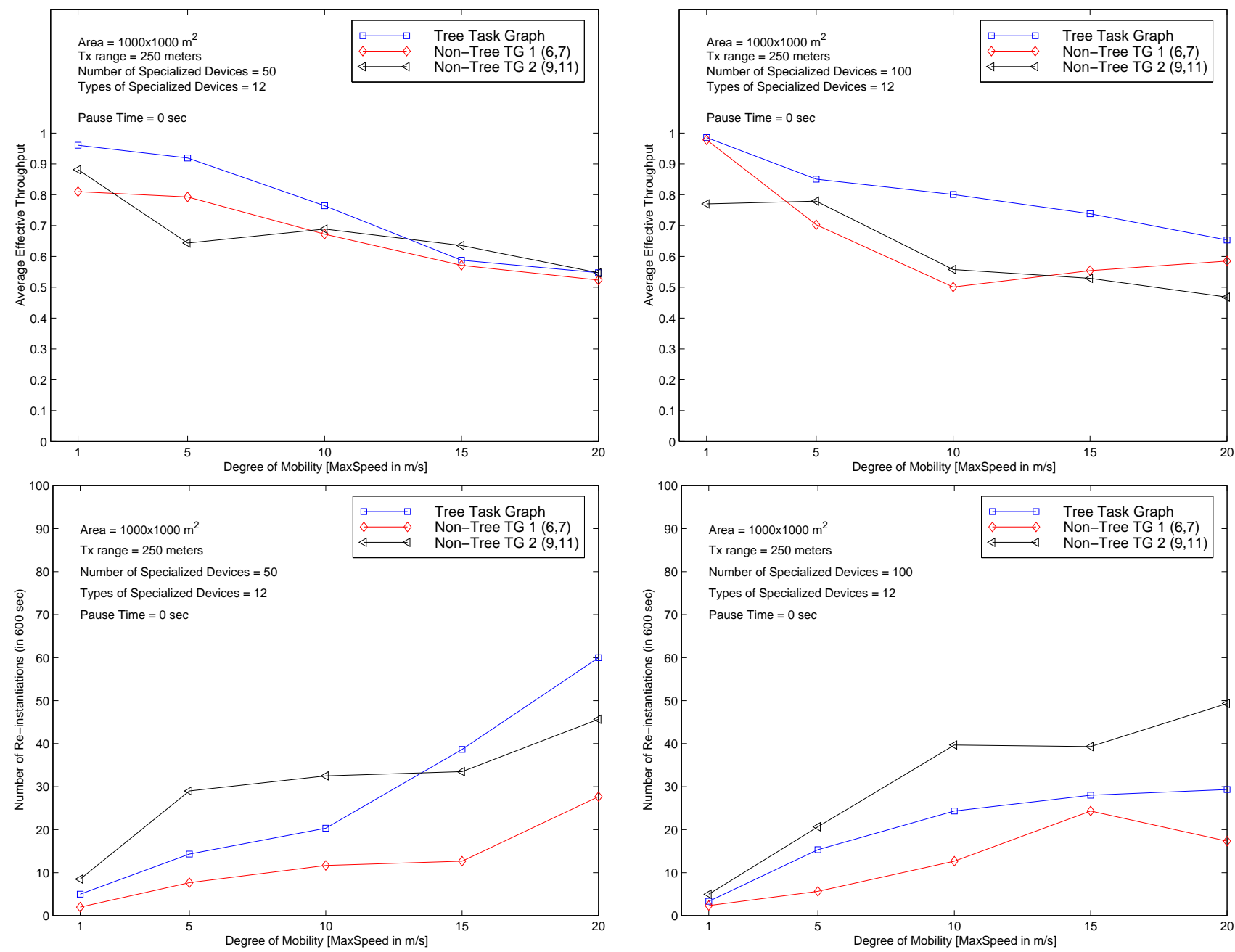

Figure 13: Medium Load scenario: Variation of Speed

throughput can be attributed to the disconnections between non-BFS parent and children which happen more often at such speeds, since the paths are likely to be longer for non-BFS edges, on average. We believe that these disconnection effects are more pronounced in dense MANETs because of the following interesting phenomenon: in a dense network, moderatehigh speeds cause frequent route errors without disconnecting the network. Since DSR uses caching, source routing and ring-zero search ${ }^{14}$, the routes contained in each device's cache become stale at high speeds. Stale routes trigger route errors which in turn trigger a route recovery mechanism at the source. For high density MANETs, more extensive route caching is likely at intermediate nodes with presence of more alternate routes. This may result in multiple unsuccessful attempts before a route discovery is successful for these longer paths which non-BFS edges have been mapped to. When this route caching/recovery mechanism

\footnotetext{
${ }^{14} \mathrm{~A}$ device requesting routes initially queries its 1-hop neighbors, and these neighbors can answer with routes in their caches.
} 

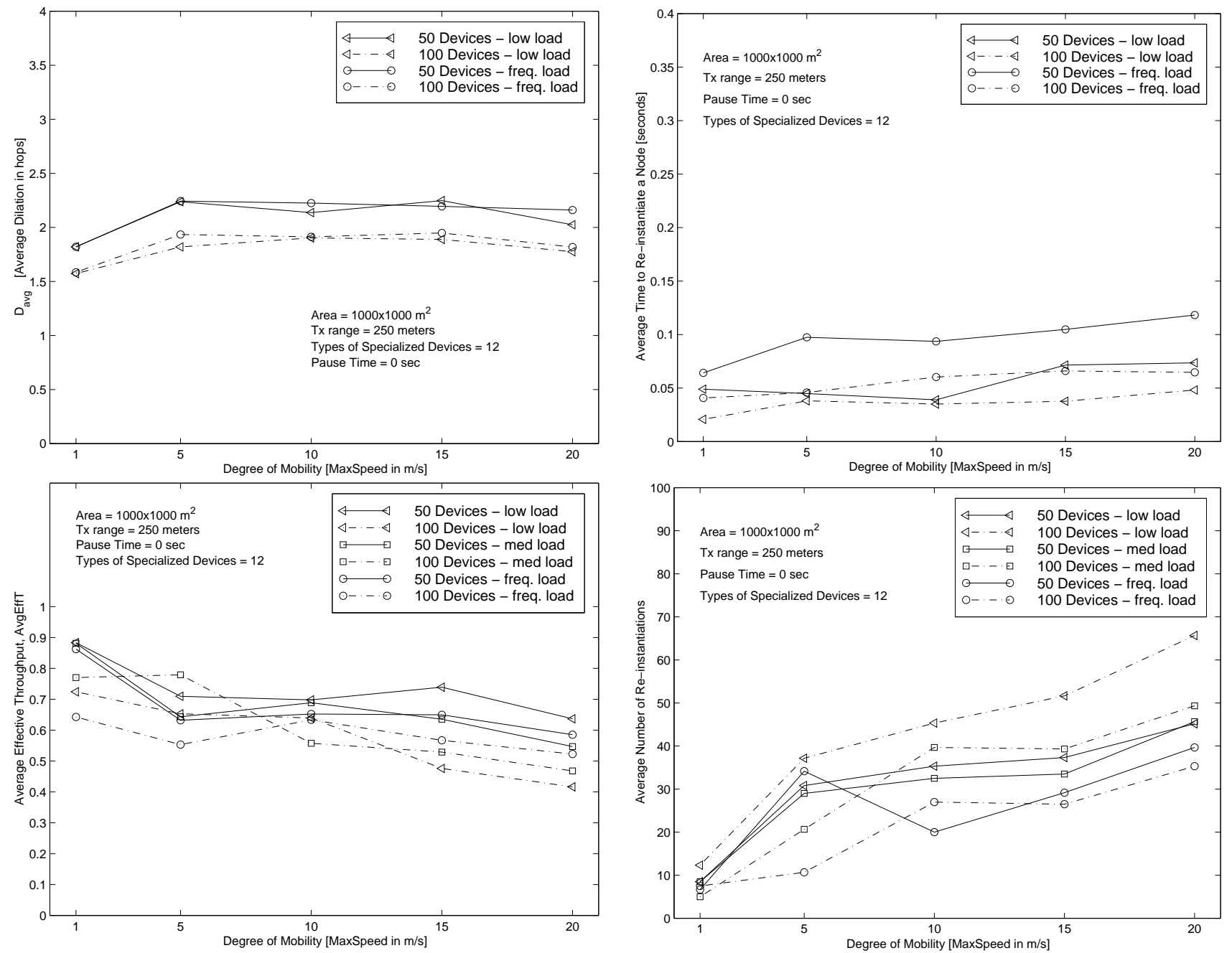

Figure 14: Effect of Variation in Rate and Granularity of Traffic for TG-2

is coupled with the TCP back-off mechanism, which assumes congestion rather than route updates, it results in longer delays in packet delivery. Since our protocol requires periodic exchange of HELLO messages over TCP, a lost HELLO packet triggers re-instantiation.

Effect of Variation in Rate and Granularity of Traffic We now examine the effect of variation in rate and granularity of traffic on the metrics for task graph TG-2 in Fig. 14. We plot both 50 and 100 device cases for different load patterns on the same scale. The parameters for the low load and frequent medium load patterns are given in Table 2. A maximum aggregate throughput of $300 \mathrm{kbps}$ can be reached in the frequent-medium scenario assuming simultaneous transmission at all instantiated devices in accordance with the underlying tuple architecture. 

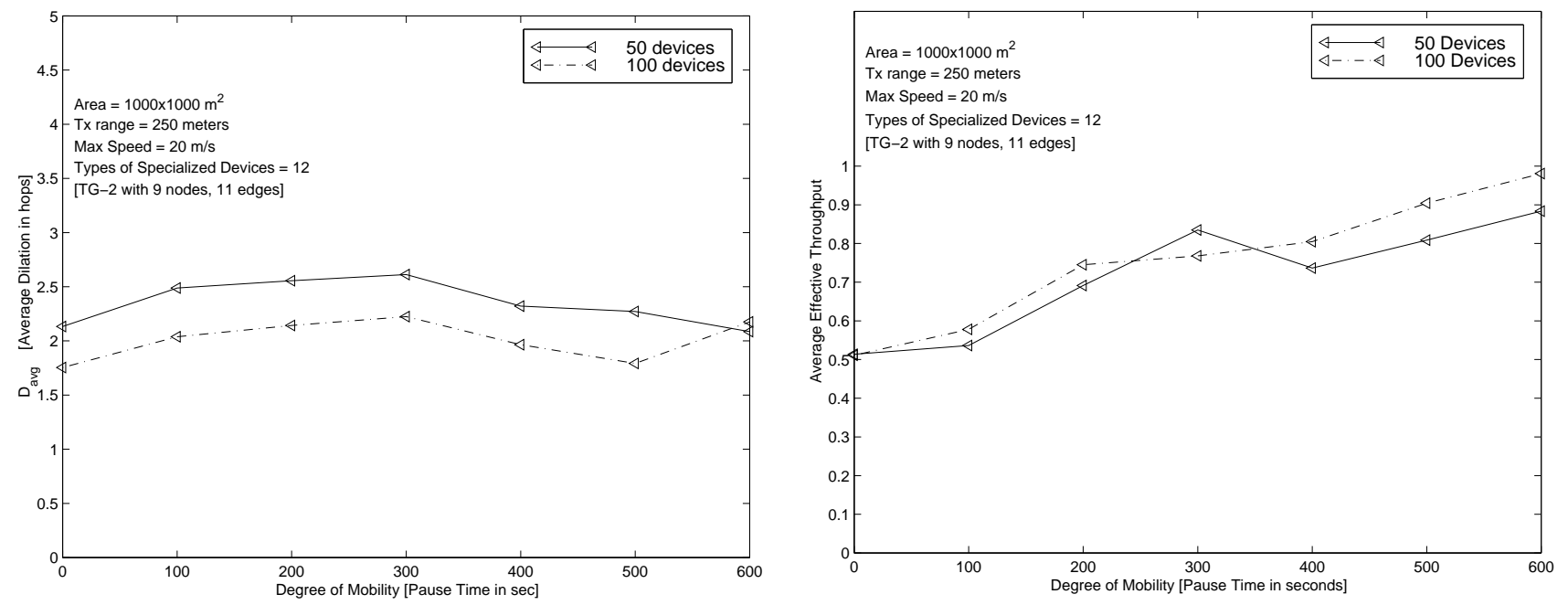

Figure 15: Effect of Varying Pause Time $((B, P)=(2500,1))$

The average dilation results (Fig. 14(a)) are similar to the medium load case, as expected - we can observe that $D_{\text {avg }}$ is impacted mainly by device density and not by the traffic rate. Next, we consider the amount of time taken to discover and re-instantiate a single node under disruption. From Fig. 14(b) we can observe that the higher density scenario has better performance for a given load type. A larger number of candidate devices in the environment increases the likelihood of finding another suitable device once the existing one is disconnected. However, we observe that the average re-instantiation times are higher for frequent-medium than those in the low load scenario. The 50 device MANET suffers more because of the compounded effect of sparse density (which means more hops) and more frequent transmission of data packets in the neighborhood resulting in more contention and hence delays.

Fig. 14(c) plots the AvgEffT curves for all three sets of load patterns. We can see that for the 50 device MANET, AvgEffT is consistently lower for medium and frequentmedium traffic loads than for low loads. On the other hand, for 100 device MANETs, throughput improves as traffic load becomes frequent for higher speeds. This can be attributed to the following reason: for dense MANETs, the route caches in the neighborhood are much fresher for frequent-medium and medium loads than for low loads. When speeds are high, route errors and repeated cache misses become dominant factors in determining effective throughput - frequent loads prevent the route caches from getting stale and thus help in quicker recovery from route errors, and hence AvgEffT improves. This can also be observed from Fig. 14(d) which demonstrates a significantly less number of re-instantiations for frequent-medium and medium loads than low loads for the 100 device MANET. 

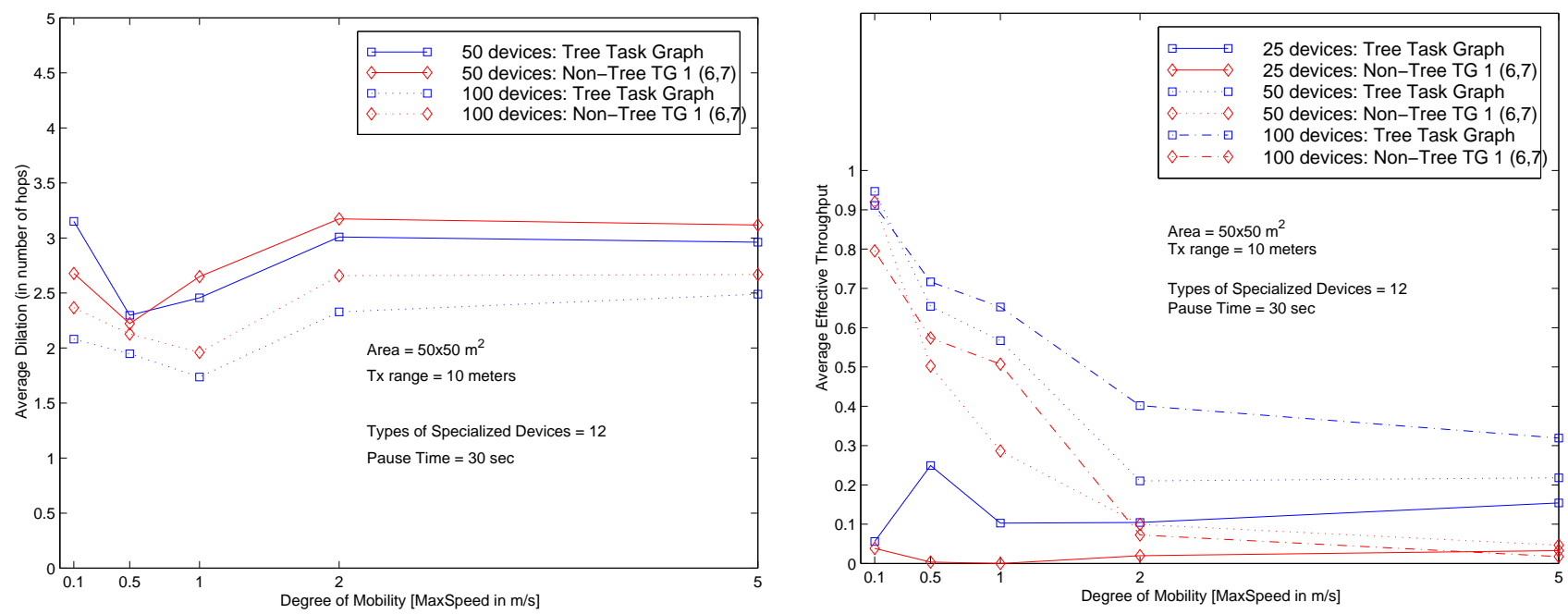

Figure 16: Short Tx range scenario: (a) Dilation, (b) AvgEffT

Effect of Variation in Pause Times Now we investigate the effect of variation of pause times (PT), which is the amount of time a device remains static after completing a random movement. The maximum speed is kept constant at $20 \mathrm{~m} / \mathrm{s}$ and data flow parameters are: $(B, P)=(2500,1)$. The results have been shown in Fig. 15. We observe that the dilation increases very gradually when mobility of the MANET reduces, and then after a certain PT, it gradually falls when the MANET becomes increasingly static. For the static case $(P T=600 s)$, the devices in the MANET are uniformly distributed and $D_{\text {avg }}$ does not change much due to re-instantiations. For the constant mobility case $(P T=0 s)$, re-instantiations happen frequently but because of the uniform density of devices in the neighborhood, a configuration with low $D_{\text {avg }}$ is discovered most of the time. However, for intermediate levels of mobility, re-instantiations do not happen that often and infrequent, non-simultaneous mobility of devices often stretches the routes which increases $D_{\text {avg }}$ slightly.

We also observe an expected phenomenon of AvgEffT increasing (almost steadily) from 0.5 to almost 1.0 when PT increases from $0 s$ to $600 \mathrm{~s}$. The denser MANET is observed to yield slightly better performance for this scenario as opposed to the lower speed/constant mobility scenarios that we have examined earlier. We attribute the reason for this to the fact that in denser, static MANETs, shorter paths are needed to communicate between two devices (as reflected by a lower value of $D_{\text {avg }}$ too), and there is a lower frequency of route errors which results in lesser packet loss.

๑With uniform probability distribution 


\begin{tabular}{|l|l|}
\hline Simulation Parameter & Value \\
\hline \hline Number of Devices & $25,50,100$ \\
\hline Simulation Area & $50 \mathrm{~m} \times 50 \mathrm{~m}$ \\
\hline Transmission Radius & $10 \mathrm{~m}$ \\
\hline Mobility Model & Random Waypoint \\
\hline MANET Routing & DSR \\
\hline \#Classes of Devices & $12^{q}$ \\
\hline Size of TG: $\left(\left|V_{T}\right|,\left|E_{T}\right|\right)$ & $(6,5),(6,7)$ \\
\hline Simulation Time & $600 \mathrm{~s}$ \\
\hline MaxSpeed & $0.1-5 \mathrm{~m} / \mathrm{s}$ \\
\hline Pause Time & $30 \mathrm{~s}$ \\
\hline Data Rate at Source $U$ & $(12500,5):$ medium \\
\hline
\end{tabular}

Table 3: Simulation Parameters for Short Transmission Range Scenario

\subsection{A Short Range Communication Scenario}

Next, we evaluate the performance of our embedding protocol in a scenario with a lower radio transmission range. Essentially we are trying to model a reasonably sized hall full of occupants carrying specialized devices that are equipped with short range radios. The simulation parameters have been listed in Table 3. The five different speeds correspond to very slow walking, walking with a normal speed, brisk walking, running, and fast running, respectively.

Fig. 16(a) shows the variation in dilation with speed of motion. We can see that the dilation is lower for the 100 device MANET than the 50 device MANET for both task graphs. This is because the network is more dense in the former case and the likelihood of finding a suitable device nearby is high. Another point to be noted is that the dilation of the TG-1 case is higher than the tree case. This is to be expected since TG-1 has extra (non-BFS) edges which are not necessarily mapped onto short paths on the physical network. One interesting observation here is that the dilation values drop when the speed is increased moderately. This happens because at moderately high speeds, the devices come closer to each other more often and that reduces the dilation. However, if the speed increases further, the associations between nearby devices are not formed due to very high relative mobility between them, and hence the dilation increases.

Fig. 16(b) shows the effect of mobility on AvgEffT. The performance for the 25 device MANET is dismal owing to the sparseness of the scenario. For very low speeds, performance in both the 50 device and 100 device MANETs is close to ideal. However, with 
gradual increase in speed, AvgE $f f T$ drops much more drastically than in the $1000 \mathrm{~m} \times 1000 \mathrm{~m}$ scenario that was considered earlier in this section. This is because the MANETs considered here are more sparse than their respective counterparts in the $1000 \mathrm{~m} \times 1000 \mathrm{~m}$ scenario, and hence the impact of increased mobility is harsher.

\section{Conclusions}

In this paper we presented a task-based framework for executing a distributed application on a network of specialized, mobile devices. We developed a task graph abstraction for applications by taking into account the dependencies induced by the data flows existing between the components of an application. We described the task-based anycasting/embedding problem and presented an optimal poly-time algorithm with respect to an average hop-count measure called dilation, for the special case where the task graph is a tree. We also described how it can be heuristically extended for general graphs. Owing to the unreasonable requirements and time complexity of the aforementioned algorithm, we presented a more practical distributed heuristic algorithm (and protocol) for anycasting a given task graph onto a MANET. We also presented a scalable, local detection and repair mechanism for recovering from task disruptions caused by mobility of devices.

We showed a plethora of simulation results for evaluating the salient properties of the proposed distributed protocol. From our simulation studies, we observed that our protocol was able to instantiate and re-instantiate TG nodes quickly with low dilation, and yielded a high effective throughput for scenarios with low mobility and reasonably high effective throughput for more mobile situations. We also noted that in some high mobility scenarios, the performance of our protocols suffered due to the non-aggressive retransmission timeout mechanism of TCP and aggressive route caching done by the underlying routing protocol, DSR. Increasing the rate and frequency of the traffic load actually helped improve the effective throughput in such situations.

In this work, we did not simulate heavy loads since standard TCP acts as a major bottleneck over multiple hops under heavy loads [14]. Our focus in this work was to demonstrate the viability of the proposed dynamic task-based anycasting approach in MANETs using existing routing and transport protocols. We believe that much superior task execution throughput can be achieved if standard TCP is replaced by feedback based schemes such as the one proposed in [8] - our future efforts will be directed towards such endeavors. Although we do not focus on reliable task execution in this paper, we note that it can be 
achieved with buffering and re-transmissions in the application.

\section{References}

[1] W. Adjie-Winoto, E. Schwartz, H. Balakrishnan, and J. Lilley, "The design and implementation of an intentional naming system," Proc. 17th ACM SOSP, Kiawah Island, SC, December 1999.

[2] G. Banavar, J. Beck, E. Gluzberg, J. Munson, J. Sussman, and D. Zukowski, "Challenges: An Application Model for Pervasive Computing," Proc. 6th ACM MobiCom, Boston, MA, August 2000.

[3] J. Beck, A. Gefflaut, and N. Islam, "MOCA: A Service Framework for Mobile Computing Devices," Proc. ACM MobiDE, Seattle, WA, August 1999.

[4] V. Bharghavan, A. Demers, S. Shenker, and L. Zhang, "MACAW: a media access protocol for wireless LAN's," Proc. SIGCOMM '94: Conference on Communications Architectures, Protocols and Applications, London, England, September 1994, pp. 212-225.

[5] Bluetooth SIG, http://www.bluetooth.com

[6] S. H. Bokhari, "On the Mapping Problem," IEEE Trans. on Computers, Vol. 30, No. 3, 1981, pp. 207-214.

[7] J. Broch, D. A. Maltz, D. B. Johnson, Y. C. Hu and J. Jetcheva, "A Performance Comparison of Multi-Hop Ad Hoc Network Routing Protocols", Proc. 4th ACM MobiCom, Dallas, TX, 1998.

[8] K. Chandran, S. Raghunathan, S. Venkatesan, and R. Prakash, "A Feedback-Based Scheme for Improving TCP Performance in Ad Hoc Wireless Networks," IEEE Personal Communications Magazine, February 2001.

[9] B. P. Crow, I. Widjaja, J. G. Kim, P. T. Sakai, "IEEE 802.11 wireless local area networks," IEEE Communications Magazine, Vol. 35, No. 9, September 1997, pp. 116126.

[10] M. Esler, J. Hightower, T. Anderson, and G. Borriello, "Next Century Challenges: DataCentric Networking for Invisible Computing The Portolano Project at the University of Washington," Proc. 5th ACM MobiCom, Seattle, WA, August 1999. 
[11] D. Estrin, R. Govindan, J. Heidemann, and S. Kumar, "Next Century Challenges: Scalable Coordination in Sensor Networks," Proc. 5th ACM MobiCom, Seattle, WA, August 1999.

[12] M. Gerla and J. T.-C. Tsai, "Multicluster, mobile multimedia radio network," Wireless Networks 1, 1995, pp. 255-265.

[13] E. Guttman, "Service Location Protocol: Automatic Discovery of IP Network Services", IEEE Internet Computing, July 1999.

[14] G. Holland and N. Vaidya, "Analysis of TCP Performance over Mobile Ad Hoc Networks," Proc. 5th ACM MobiCom, pp. 219-230, Seattle WA, August 1999.

[15] P. Jacquet, P. Muhlethaler, A. Qayyum, A. Laouiti, L. Viennot, and T. Clausen, "Optimized Link State Routing Protocol," Internet-Draft, draft-ietf-manet-olsr-04.txt, September 2001. Work in Progress.

[16] T. Hodes, R. Katz, E. Servan-Screiber, and L. Rowe, "Composable Ad-Hoc Mobile Services for Universal Interaction," Proc. 3rd ACM MobiCom, 1997.

[17] D. B. Johnson and D. A. Maltz, "Dynamic Source Routing in Ad Hoc Wireless Networks", in Mobile Computing, edited by Tomasz Imielinski and Hank Korth, chapter 5, pages 153-181, Kluwer Academic Publishers, 1996.

[18] P. Karn, "MACA - A new channel access method for packet radio," Proc. 9th ARRL/CRRL Amateur Radio Computer Networking Conference, September 1990, pp. 134-140.

[19] R. Monien and H. Sudborough, "Embedding one Interconnection Network in Another," Computing Suppl. 7, 1990, pp. 257-282.

[20] C. E. Perkins, E. M. Royer, and S. R. Das, "Ad Hoc On-Demand Distance Vector (AODV) Routing," Internet-Draft, draft-ietf-manet-aodv-08.txt, March 2001. Work in Progress.

[21] Sun Microsystems, "Jini Technology Core Platform Specification," http://www.sun.com/jini/specs

[22] VINT Network Simulator - ns (version 2). http://www.isi.edu/nsnam/ns/ 